\title{
LIE TRANSFORMATION GROUPS
}

by

JAMES P. THOMSON

B.Sc., University of British Columbia; 1972

A THESIS SUBMITTED IN PARTIAL FULFILMENT OF THE REQUIREMENTS FOR THE DEGREE OF

MASTER OF SCIENCE

in the Department

of

MATHEMATICS

We accept this thesis as conforming to

the required standard

THE UNIVERSITY OF BRITISH COLUMBIA

October, 1974 
In presenting this thesis in partial fulfilment of the requirements for an advanced degree at the University of British Columbia, I agree that the Library shall make it freely available for reference and study. 1 further agree that pemission for extensive copying of this thesis for scholarly purposes may be granted by the Head of my Department or by his representatives. It is understood that copying or publication of this thesis for financial gain shall not be allowed without my written pemission.

Department of Mathematics

The University of British Columbia Vancouver 8 , Canada

Date Nov. 25, 1974 


\section{ABSTRACT}

Suppose $G$ is a Lie group and $M$ is a manifold $(G$ and $M$ are not necessarily finite dimensional). Let $D(M)$ denote the group of diffeomorphisms on $M$ and $V(M)$ denote the Lie algebra of vector fields on $M$. If $X$ is a complete vector field then Exp tX will denote the one-parameter group of $X$. A local action $\phi$ of $G$ on $M$ gives rise to a Lie algebra homomorphism. $\phi^{+}$from $L(G)$ into $V(M)$. In particular if $G$ is a subgroup of $D(M)$ and $\phi: G \times M \rightarrow M$ is the natural global action $(g, p) \rightarrow g .(p)$ then $G$ is called a Lie transformation group of $M$. If $M$ is a Hausdorff manifold and $G$ is a Lie transformation group of $M$ we show that $\phi^{+}$is an isomorphism of $L(G)$ onto $\phi^{+}(L(G))$ and $L=\phi^{+}(L(G))$ satisfies the following conditions :

(A) L consists of complete vector fields.

(B) L has a Banach Lie algebra structure satisfying the following two conditions :

(B1) the evaluation map ev $:(X, p) \rightarrow X(p)$ is a vector bundle morphism from the trivial bundle $\mathrm{L} \times \mathrm{M}$ into $\mathrm{T}(\mathrm{M})$,

(B2) there exists an open ball $\mathrm{B}_{\mathrm{r}}(0)$ of radius $r$ at 0 such that $\operatorname{Exp}: \mathrm{L} \longrightarrow \mathrm{D}(\mathrm{M})$ is injective on $\mathrm{B}_{\mathbf{r}}(0)$.

Conversely, if $L$ is a subalgebra of $V(M)$ (M Hausdorff) satisfying conditions (A) and (B) we show there exists a unique connected Lie transformation group with natural action $\phi: G \times M \longrightarrow M$ such that $\phi^{+}$is a Banach Lie algebra isomorphism of $L(G)$ onto $L$. 


\section{Table of Contents}

Page

Chapter 1

Preliminaries

8.1. Foliations and Integrabies Subbund1es; 1

§2 Total Differential Equations 3

§3 Lie Groups and Lie Algebras.

Chapter 2

Local and Infinitesimal Group Actions

\$4. Local Group Actions 99

$\$ 5$ Infinitesimal Actions $\quad 13$

§6. The Infinitesimal Graph $\quad 17$

§7 Existence Theorem $\quad 21$

§8 Uniform Infinitesimal Actions 23

Chapter 3

Connected Lie Transformation Groups

\$9 The Image of the Infinitesimal Generator of a Lie Transformation Group $\quad 30$

\$10 Banach Lie Algebras of Complete Vector Fields 33

\$11 A Banach Lie Algebra of Complete Vector Fields which does not generate a Connected Lie Transformation Group 


\section{Acknowledgements}

I wish to thank Dr. Ottmar Loos for the suggestion of this thesis topic and the many helpful suggestions and criticisms throughout its development. I would also like to acknowledge Dr. Jens Gamst who initially generated : my enthusiasm for differential geometry..

Appreciation is expressed to the Department of Mathematics for providing financial support during the period of my graduate studies at the University of British Columbia. 
Chapter 1

\section{Preliminaries}

Al1 manifolds considered are real Banach manifolds of class $\mathrm{C}^{\mathrm{K}}$ where $\mathrm{K}=\infty$ or $\mathrm{K}=\omega$. The word morphism will mean a $\mathrm{C}^{\mathrm{K}}$ map between $\mathrm{C}^{\mathrm{K}}$ manifolds. In this chapter, we collect the necessary facts on foliations of manifolds and on infinite dimensional Lie groups. Almost all of this material will come from Bourbaki [1, \$9] or Bourbaki $[2$, Chapter 3$]$

\section{\$1 Foliations and Integrable Subbundles}

Let $M$ and $S$ be manifolds and $p: M \longrightarrow S$ a submersion. We then have, for each $s \in S$, a manifold structure induced on the level set $\mathrm{p}^{-1}(\mathrm{~s})$ by $\mathrm{M}$. Denote by $\mathrm{M}_{\mathrm{p}}$ the manifold which is the disjoint union over $s$ of $p^{-1}(s)$. Each $p^{-1}(s)$ is an open submanifold of $M_{p}$ and topologically $M_{p}$ is the topological sum of the topological spaces $p^{-1}(s)$.

Definition (1.1) Let $M$ be a manifold. A foliation of $M$ is a manifold $Y$ having the same point set as $M$ and satisfying the condition that for all $x \in M$, there exists an open submanifold $U$ of $M$ containing $x$, a manifold $S$, and a submersion $\mathrm{p}: \mathrm{U} \longrightarrow \mathrm{S}$ such that the manifold $\mathrm{U}_{\mathrm{p}}$ is an open submanifold of $\mathrm{Y}$..

The inclusion map of $Y$ into $M$ is easily seen to be a bijective 
immersion.

We call the pair (M, Y) a foliated manifold. A set. $U$ is called a (connected) 1eaf if it is a (connected) open set in $Y$. The maximal connected leaves are therefore the connected components of $Y$.

Definition (1.2) If $(M, Y)$ and $\left(M^{\prime}, Y^{\prime}\right)$ are foliated manifolds, a morphism from $(M, Y)$ into $\left(M^{\prime}, Y^{\prime}\right)$ is a map which is a morphism of $M$ into $M^{\prime}$ and at the same time a morphism of $Y$ into $Y^{\prime}$.

Using the inclusion map of $Y$ into $M$, for each $x \in M$ we can identify the tangent space $T_{X}(Y)$ with a subspace of $T_{X}(M)$. With this identification we have the following propositions.

Proposition (1.3) The spaces $\mathrm{T}_{\mathrm{X}}(\mathrm{Y})$ are the fibers of a subbundle $\mathrm{T}(\mathrm{M}, \mathrm{Y})$ of $\mathrm{T}(\mathrm{M})$. Furthermore if $\mathrm{Y}$ is defined by a submersion $\mathrm{P}: \mathrm{M} \rightarrow \mathrm{S}$, then $T(M, Y)=\operatorname{ker} T(p)$.

Proposition (1.4) Let $(M, Y)$ and $\left(M^{\prime}, Y^{\prime}\right)$ be two foliated manifolds and $f: M \rightarrow M^{\prime}$ be a morphism. A necessary and sufficient condition that $f$ is a morphism from $(M, Y)$ into ( $\left.M^{\prime}, Y^{\prime}\right)$ is that $T(f)$ takes $T(M, Y)$ into $T\left(M^{\prime}, Y^{\prime}\right)$.

Let. $F$ be a subbundle of $T(M)$. We now examine the conditions on $F$ which imply the existence of a manifold $Y$ such that $T(M, Y)=F$. If this is the case then $F$ is called an integrable subbundle of $T(M)$ and the foliation it defines is unique. 
Theorem of Frobenius (1.5) $F$ is integrable if there exists a family

$\left\{\xi_{i}\right\}_{i \varepsilon I}$ of sections of $F$ such that

(1) for a11 $x \in M$ the set $\left\{\xi_{i}(x): i \varepsilon I\right\}$ is a total subset

of the fiber $F_{x}$ of $F$ above $x$.

(2) for al1 pairs ( $i, j)$ of elements of $I$ andali $x \in M$,

$\left[\xi_{i}, \xi_{j}\right](x) \varepsilon \cdot \dot{F}_{i x}$

\$2 Tota1 Differential Equations

We now construct a particular subbundle and examine what it means for it to be integrable. This will be the setting for discussing generalized differential equations.

Suppose $M$ is the product of two manifolds $A$ and $B$. Let $\mathrm{p}_{1}: \mathrm{M} \longrightarrow \mathrm{A}$ and $\mathrm{p}_{2}: \mathrm{M} \longrightarrow \mathrm{B}$ be the projections on the first and second factors. There are two subbundles, $\mathrm{p}_{1} * \mathrm{~T}(\mathrm{~A})$ and $\mathrm{p}_{2} * \mathrm{~T}(\mathrm{~B})$, of $T(M)=T(A) \dot{x} T(B)$ associated with $\mathrm{p}_{1}$ and $\mathrm{p}_{2}$. The fiber $\mathrm{p}_{1}^{* \mathrm{~T}(\mathrm{~A})}(\mathrm{a}, \mathrm{b})$ of $\mathrm{p}_{1} * \mathrm{~T}(\mathrm{~A})$ over $(\mathrm{a}, \mathrm{b})$ is $\mathrm{T}_{a}(\mathrm{~A}) \times\left\{\mathrm{O}_{\mathrm{b}}\right\}$ where $\mathrm{O}_{\mathrm{b}}$ is the zero vector in $\mathrm{T}_{\mathrm{b}}(\mathrm{B})$. We identify this fiber with $\mathrm{T}_{\mathrm{a}}(\mathrm{A})$. Similarly the fiber $\mathrm{p}_{2}{ }^{* \mathrm{~T}(\mathrm{~B})}(\mathrm{a}, \mathrm{b})$ of $\mathrm{p}_{2}{ }^{* \mathrm{~T}(\mathrm{~B})}$ over $(\mathrm{a}, \mathrm{b})$ is $\left\{\mathrm{O}_{\mathrm{a}}\right\} \times \mathrm{T}_{\mathrm{b}}(\mathrm{B})$ which is identified with $\mathrm{T}_{\mathrm{b}}(\mathrm{B})$.

Let $f$ be a vector bundle morphism from $\mathrm{p}_{1} * \mathrm{~T}(\mathrm{~A})$ into $\mathrm{p}_{2}{ }^{* \mathrm{~T}}(\mathrm{~B})$. Then for each $(a, b) \varepsilon M ; f$ is a continuous linear map $\mathrm{f}_{(\mathrm{a}, \mathrm{b})}: \mathrm{T}_{\mathrm{a}}(\mathrm{A}) \rightarrow \mathrm{T}_{\mathrm{b}}(\mathrm{B})$ (after identifying $\mathrm{T}_{\mathrm{a}}(\mathrm{A})$ with $\mathrm{p}_{1}{ }^{*} \mathrm{~T}(\mathrm{~A})(\mathrm{a}, \mathrm{b})$ 
and $T_{b}(B)$ with $\left.p_{2}{ }^{*} T(B)(a, b)\right)$.

Proposition (2.1) The graphs of the $f_{(a, b)}$ are the fibers of a subbundle of $\mathrm{T}(\mathrm{M})$ which we denote by $\mathrm{F}^{\mathrm{f}}$.

Definition (2.2) Let $A^{\prime}$ be an open set in $A$. A morphism $\phi: A^{\prime} \longrightarrow B$ is called an integral of $f$ if for all a. $\varepsilon A^{\prime}$ one has $T_{a}(\phi)=f(a, \phi(a))^{\bullet}$

The following two propositions describe the local uniqueness of integrals.

Proposition (2.3) If $\phi_{1}$ and $\phi_{2}$ are two integrals of $f$ taking the same value at a point a $\varepsilon A$, then they coincide in a neighbourhood of $a$.

Proposition (2.4) Let $\mathrm{Z}$ be a manifold, $\mathrm{A}^{\prime}$, an open set in $\mathrm{A}$, and a $\varepsilon A^{\prime}$. Suppose $\phi_{1}$ and $\phi_{2}$ are morphisms of $Z \times A^{\prime}$ into $B$ such that $\phi_{1}$ and $\phi_{2}$ coincide on $z \times\{a\}$ and for a11. $z \varepsilon z$, the morphisms $a \longrightarrow \phi_{1}(z, a)$ and $a \longrightarrow \phi_{2}(z, a)$ are integrals of $f$. Then $\phi_{1}$ and $\phi_{2}$ coincide on a neighbourhood of $Z \times\{a\}$.

Suppose now that $\mathrm{F}^{\mathrm{f}}$ is integrable and therefore defines a foliation $Y$ of $M$ with $T(M, Y)=F^{f}$. Let $\phi: A^{\prime} \longrightarrow B$ be an integral for $f$ and define $\psi: A^{\prime} \longrightarrow M$ by $\psi(a)=(a, \phi(a))$. We have $\mathrm{T} \psi\left(\mathrm{T}\left(\mathrm{A}^{\prime}\right)\right) \subset \mathrm{F}^{\mathrm{f}}$ since $\phi$ was an integral and Proposition (1.4) gives that $\psi$ is also a morphism from $A^{\prime}$ into $Y$. Let $v_{a} \in T_{a} A^{\prime}$. Now $T_{a} \psi\left(v_{a}\right)=\left(v_{a}, T_{a} \phi\left(v_{a}\right)\right)=\left(v_{a}, f_{(a, \phi(a))}\left(v_{a}\right)\right)$ which implies $T_{a} \psi$ is an 
isomorphism (of Banach spaces) of $\mathrm{T}_{\mathrm{a}} \mathrm{A}^{\prime}$ onto $\mathrm{F}^{\mathrm{f}}(\mathrm{a} ; \phi(a))$. This means $\psi$ is a local diffeomorphism into $\mathrm{Y}$ at $\mathrm{a}$ and as $\mathrm{a}$ was arbitrary we have proven the following result.

Proposition $(2.5)$ If $\mathrm{F}^{\mathrm{f}}$ is integrable and $\phi: \mathrm{A}^{\prime} \rightarrow \mathrm{M}$ is an integral for $f$ then $\left\{(a, \phi(a)): a \in A^{\prime}\right\}$ is a leaf (open set) of the foliation defined by $\mathrm{F}^{\mathrm{f}}$.

We complete this section with the existence theorem for integrals.

Proposition (2.6) Suppose that $\mathrm{F}^{\mathrm{f}}$ is integrable. Let $\left(z_{0}, a_{0}\right) \varepsilon \mathrm{Z} \times \mathrm{A}$ and $\rho$ be a morphism from $Z$ into B : Then there exists an open neighbourhood $Z^{\prime} \times A^{i}$ of $\left(z_{0}, a_{0}\right)$ in $Z \times A$ and a morphism $\phi: Z^{\prime} \times A^{\prime} \longrightarrow B$ such that for every $z \in Z^{\prime}$ the morphism $a \longrightarrow \phi(z, a)$ of $A^{\prime}$ into $B$ is an integral for $f$ and $\rho(z)=\phi\left(z, a_{0}\right)$.

We will mainly use this with $Z=B$ and $\rho=$ identity.

\section{\$3 Lie Groups and Lie Algebras}

A Lie group $G$ is a group, which is also a Banach manifold (not necessarily finite dimensional) such that the operations of multiplication $G \times G \longrightarrow G$ and taking inverses $G \longrightarrow G$ are morphisms. $G$ will be called finite (infinite) dimensional if its manifold structure is modelled on a finite (infinite) dimensional Banach space. 
A Banach Lie Algebra $L$ is a Lie algebra with a Banach space structure such that the bracket $[]:, L \times L \rightarrow L$ is continuous. We cal1 L finite (infinite) dimensional if the underlying vector space is finite (infinite) dimensional.

Almost all of the standard finite dimensional Lie group theory carries over to infinite dimensions. If $G$ is a Lie group then there is a Banach Lie algebra $L(G)$ corresponding to $G$ and an exponential map from $L(G)$ into $G$, which is a local diffeomorphism at 0 . (We break with the usual convention of having $L(G)$ equal to the set of left invariant vector fields on $G$ and instead it will be the set of right invariant vector fields. Defining $L(G)$ to be the right invariant vector fields will make the definition of an infinitesimal action in Chapter 2 easier. This is a slight change since if we identify $L(G)$ with $T_{e}(G)$, the tangent space at the identity, then the only difference between the right invariant Lie algebra structure and left invariant Lie algebra structure is that the bracket differs by a sign.)

The major difference between the finite and infinite dimensional theories is that there exist infinite dimensional Banach Lie algebras $\mathrm{L}$ for which there does not exist any Lie group $G$ such that $L=L(G)$. If a Lie group $G$ does exist such that $L=L(G)$ then the Banach Lie algebra $\mathrm{L}$ is called enlargeable. For an example of a non-enlargeable Banach Lie algebra see Est and Korthagen [4]: Although L may not be enlargeable, a Banach Lie algebra closely related to $L$ is always enlargeable. This Lie algebra is the path space of $L$ which we now examine. 
Let $B L$ denote the category of Banach Lie algebras with continuous homomorphisms as morphisms. Then we have the path functor $\Lambda: B L \rightarrow B L$ which takes $\mathrm{L}$ to $\Lambda \mathrm{L}=[\mathrm{f} \mid \mathrm{f}:[0,1] \rightarrow \mathrm{L}$ continuous with $f(0)=0\}$ with the following Lie algebra structure. If $\mathrm{f}, \mathrm{g} \varepsilon \Lambda \mathrm{L}$ then the norm of $f$ is $\max _{t \in[0,1]}\|f(t)\|$ and the bracket is defined pointwise, $[f, g](t) \equiv[f(t), g(t)]$. If $\phi: L \rightarrow L^{\prime}$ is a morphism of Banach Lie algebras then $\Lambda \phi: \Lambda \mathrm{L} \longrightarrow \mathrm{L}^{\prime}$ is given by $\Lambda \phi(\mathrm{f})=\phi \circ \mathrm{f}$.

Theorem (3.1) Let $L$ be a Lie algebra and $\Lambda \mathrm{L}$ be as above. Then

(1) the endpoint evaluation map $f \longrightarrow f(1)$ from $\Lambda L$ into $L$ is continuous.

(2) $\Lambda \mathrm{L}$ is enlargeable.

Proof : The proof of (1) is obvious from the definition of $\Lambda L$. The reader is referred to Swierczkowski [8] for a proof of (2).

For later reference, we now list some facts on subgroups and subalgebras of Lie groups and Banach Lie algebras. The proofs are in Bourbaki [2, Chapter 3].

Definition (3.2) A subset $H$ of $G$ is a Lie subgroup of $G$ if it is a subgroup and a submanifold of $G$.

Proposition (3.3) Let $\mathrm{H}$ be a subgroup of a Lie group $G$. A necessary and sufficient condition for $H$ to be a Lie subgroup is that there exists a point $h \in H$ and an open neighbourhood $U$ of $h$ in $G$ such that 
$H \cap U$ is a submanifold of $G$.

Let $L$ be a Banach Lie algebra. A Banach Lie subalgebra of $L$ is a closed vector subspace of $I$ which is closed under. the bracket operation, i.e. a subalgebra. If $\mathrm{H}$ is a lie subgroup of a Lie group $G$ then using the inclusion we identify $L(H)$ with a Banach Lie subalgebra of $L(G)$ which splits in $L(G)$. (A closed subspace $F$ of a Banach space $E$ is said to split if there exists a closed subspace $F_{1}$ such that $\mathrm{F}+\mathrm{F}_{1}=\mathrm{E}$ and $\mathrm{F} \cap \mathrm{F}_{1}=0$ ). If in addition $\mathrm{H}$ is normal then $\mathrm{L}(\mathrm{H})$ is an ideal in $L(G)$, i.e. $[L(G), L(H)] \subset L(H)$.

Proposition (3.4) Let $G$ be a Lie group and $\mathrm{H}$ be a norma1 Lie subgroup of G. Then there exists.a structure of a Lie group on G/H such that the projection map is a submersion and $L(G / H) \cong L(G) / L(H)$.

Proof : Bourbaki [2, prop. 11, p.105 and p.141] 
Chapter 2

\section{Local and Infinitesimal Group Actions}

We determine the correspondence between local group actions and infinitesimal group actions in thîs chapter. Our treatment of this subject follows that of Palais [7].

Before proceeding we establish some notation conventions: G will denote a connected Lie group and $L(G)$ will be its Banach Lie algebra of right invariants vector fields. Right multiplication by an element $g \in G$ will be denoted by $R(g)$. The identity element in $G$ will be denoted by e. $M$ will denote a manifold and $V(M)$ will be the Lie algebra of vector fields on $M$.

\section{$\$ 4$ Local Group Actions}

Definition (4.1) A local (1eft) action of $G$ on $M$ is a morphism $\phi$ from an open set $D$ containing $\{e\} \times M$ in $G \times M$ into $M$ satisfying the following conditions :

(1) $\phi(e, p)=p$ for a11 $p \in M$.

(2) If $(h, p),(g, \phi(h, p))$ and (gh, p) all belong to $D$ then $\phi(g h, p)=\phi(g, \phi(h, p))$.

If $D=G \times M$ then $\phi$ is called a global action of $G$ on $M$. 
Let $D^{p}=\{g:(g, p) \varepsilon D\}$. The morphism $g \rightarrow \phi(g, p)$ of $\mathrm{D}^{\mathrm{P}}$ into $\mathrm{M}$ will be denoted by $\phi^{\mathrm{P}}$.

The definition of local action we have given is from Palais. [7]. Bourbaki [2, p.118] gives what appears to be a different definition of local action as follows.

Definition (4.1(a)) (Bourbaki) A local (left) action of $G$ on $M$ is a morphism $\psi$ defined on an open set $\Omega$ of $G \times M$ containing $\{e\} \times M$, with values in $M$, possessing the following properties

(1) $\psi(e, p)=p$ for all $p \in M$;

(2) there exists a neighbourhood $\Omega_{1}$ of $\{\mathrm{e}\} \times\{\mathrm{e}\} \times M$ in $G \times G \times M$ such that, for $\left(g, g^{\prime}, p\right) \cdot \varepsilon \Omega_{1}$, the elements $\left(g^{\prime}, p\right)$, $\left(g g^{\prime}, p\right),\left(g, \psi\left(g^{\prime}, p\right)\right)$ are in $\Omega$ and $\psi\left(g, \psi\left(g^{\prime}, p\right)\right)=\psi\left(g g^{\prime}, p\right)$.

This is slightly different from the version in Bourbaki since we aren't considering actions of "grouplets".

Proposition (4.2) Definition (4.1) and Definition (4.1(a)) are equivalent.

Proof : Def.(4.1) implies Def.(4.1(a))

Let $\psi=\phi$ and $\Omega=D$. We have to find an open set $\Omega_{1}$ in $G \times G \times M$ satisfying condition (2) in Def.(4.1(a)). Define $\delta$ from $G \times D$ into $G \times M$ by $\delta(g, h, p)=(g, \phi(h, p))$, then $\delta^{-1}(D)$ is open and contains $\{e\} \times\{e\} \times M$. Define $\gamma$ from $G \times D$ into $G \times M$ by $\gamma(g, h, p)=(g h, p)$ then $\gamma^{-1}(D)$ is open and contains $\{e\} \times\{e\} \times M$. 
Let $\Omega_{1}=\delta^{-1}(D) \cap \gamma^{-1}(D)$, then $\Omega_{1}$ is an open neighbourhood of $\{\mathrm{e}\} \times\{\mathrm{e}\} \times M$ and if $(\mathrm{g}, \mathrm{h}, \mathrm{p}) \varepsilon \Omega_{1}$ we have $(\mathrm{h}, \mathrm{p}) \varepsilon \mathrm{D} ;(\mathrm{g}, \phi(\mathrm{h}, \mathrm{p})) \varepsilon \mathrm{D}$ since $(g, h, p) \varepsilon \delta^{-1}(D)$; and $(g h, p) \varepsilon D$ since $(g, h, p) \varepsilon \gamma^{-1}(D)$. Then Def:(4.1) (2) gives

$$
\psi(g, \psi(h, p))=\phi(g, \phi(h, p))=\phi(g h, p)=\psi(g h, p)
$$

and condition (2) of Def.(4.I(a)) is satisfied.

Def.(4.1(a)) implies Def.(4.1)

Let $\phi=\psi$. We will find D such that condition (2) of Def.(4.1) is satisfied. Let $\Omega_{1}$ be as in Def.(4.1(a)). Since $\Omega_{1}$ is an open neighbourhood of $\{e\} \times\{e\} \times M$ we can find neighbourhood's $v_{p}$ and $U_{p}$ of $e$ in $G$ and $W_{p}$ of $\cdot p$ in $M$ such that $v_{p} \times U_{p} \times W_{p} \subset \Omega_{1}$. For each $p \in M$, let $G_{p}=\exp \left(B_{r}(0)\right)$ where $B_{r}(0)$ is the ball of radius $r$ centered at 0 in $L(G)$ and $r$ is so small that $G_{p}^{2} \subset V_{p} \cap U_{p}$ Then $G_{p}$ is connected, $G_{p}=G_{p}^{-1}, G_{p} \subset U_{p}, G_{p} \subset V_{p}$, and $G_{p} \times G_{p} \times W_{p}$ is an open neighbourhood of $(e, e, p)$ contained in $\Omega_{1}$. Also $\left\{G_{p}\right\}_{p \in M}$ are ordered by inclusion so if we have $G_{x}$ and $G_{y}$ then either $G_{x} \subset G_{y}$ or $G_{y} \subset G_{x}$. Define, $D=\bigcup_{p \in M} G_{p} \times W_{p}$ and suppose $(h, p),(g, \phi(h, p))$ and $(\mathrm{gh}, \mathrm{p}) \in \mathrm{D}$. Since $\mathrm{D}$ is "symmetric" (each $\mathrm{G}_{\mathrm{p}}$ was symmetric) we have $(h, p) \varepsilon D$ implies $\left(h^{-1}, p\right) \varepsilon D$. Now by the definition of $D ;$ (gh, p) and $\left(h^{-1}, p\right)$ belonging to $D$ means there exists $x \in M$ such that $\left(h^{-1}, p\right) \varepsilon G_{x} \times W_{x}$ and there exists y $\varepsilon M$ such that $(g h, p) \varepsilon G_{y} \times W_{y}$. By the remark above either $G_{x} \subset G_{y}$ or $G_{y} \subset G_{x}$ so (without loss of 
generality) assuming the latter we have $(g h, p) \varepsilon G_{x} \times W_{x}$ also. Now $\mathrm{G}_{\mathrm{x}}^{2} \subset \mathrm{V}_{\mathrm{x}} \cap \mathrm{U}_{\mathrm{x}}$ implies $\left((\mathrm{gh})\left(\mathrm{h}^{-1}\right), \mathrm{p}\right) \varepsilon \mathrm{G}_{\mathrm{x}}^{2} \times \mathrm{W}_{\mathrm{x}} \subset \mathrm{V}_{\mathrm{x}} \times \mathrm{w}_{\mathrm{x}}$, i.e. $(g, p) \varepsilon V_{x} \times W_{x}:$ We also have $(h, p) \varepsilon G_{x} \times W_{x} \subset U_{x} \times W_{x}$ which means $(\mathrm{g}, \mathrm{h}, \mathrm{p}) \in \mathrm{V}_{\mathrm{x}} \times \mathrm{U}_{\mathrm{x}} \times \mathrm{W}_{\mathrm{x}} \subset \Omega_{1}$ and condition (2) of Def.(4.I(a)) gives $\phi(g, \phi(h ;, p))=\psi(g, \psi(h+p) *=\psi \psi \psi(g h, p)=\phi(g h, p)$

Examples of local actions

Example (4.3): Let $M$ be a paracompact manifold and $\xi$ be a vector field on $M$. Then the flow (see Bourbaki $[1, \S 9]$ ) of $\xi$ is a local left action of $\mathbb{R}$ on $M$.

Example (4.4): If $E$ and $F$ are Banach spaces then denote by Hom(E, F) the Banach space of continuous linear maps from $E$ into $F$ and by GL(F) the Lie group of invertible elements in $\operatorname{Hom}(F, F)$. GL(F) is open in $\operatorname{Hom}(F, F)$. (See Lang [5, p.5] for proofs). Let $M=\operatorname{Hom}(F, E), G$ be the additive Lie group $\operatorname{Hom}(E, F)$, and $I_{F}$ be the identity in $G L(F)$. Define the morphism $\gamma: G \times M \rightarrow \operatorname{Hom}(F, F)$ by $\gamma(g, p)=g \circ p+I_{F} \cdot$ Let $\mathrm{D}=\gamma^{-1}(\mathrm{GL}(\mathrm{F}))$; then $\mathrm{D}$ is open and contains $\{0\} \times M$. Define the local action $\phi: D \longrightarrow M$ of $G$ on $M$ by $\phi(g, p)=p^{\circ}\left(g \circ p+I_{F}\right)^{-1} \cdot \phi$ is a local action for;

(1) $\phi(0, p)=p \circ\left(0+I_{F}\right)^{-1}=p$ 
(2) $\phi(g, \phi(h, p))=\phi(h, p) \circ\left(g \circ \phi(h, . p)+I_{F}\right)^{-1}$

$$
\begin{aligned}
& =p \circ\left(h \circ p+I_{F}\right)^{-1}\left(g \circ p \circ\left(h \circ p+I_{F}\right)^{-1}+I_{F}\right)^{-1} \\
& =p \circ\left(\left(g \circ p \circ\left(h \circ p+I_{F}\right)^{-1}+I_{F}\right)\left(h \circ p+I_{F}\right)\right)^{-1} \\
& =p \circ\left(g \circ p+h \circ p+I_{F}\right)^{-1} \\
& =p \circ\left((g+h) \circ p+I_{F}\right)^{-1} \\
& =\phi(g+h, p) .
\end{aligned}
$$

\section{§5 Infinitesimal Actions}

Let $\mathrm{L}$ be a Banach Lie algebra.

Definition (5.1) A (left) action of $L$ on $M$ is a Lie algebra homomorphism $\theta: L \longrightarrow V(M)$, satisfying the condition that the evaluation map $(\mathrm{x}, \mathrm{p}) \rightarrow \theta(\mathrm{x})(\mathrm{p})$ is a vector bundle morphism from the trivial vector bundle $\mathrm{L} \times \mathrm{M}$ into $\mathrm{T}(\mathrm{M})$.

Remarks : (1) If $L=L(G)$ for some Lie group $G$ then $\theta$ is called an infinitesimal (1eft) action of $G$ on $M$.

(2). If $L$ is finite dimensional then the evaluation map is automatically a vector bundle morphism (Bourbaki [2, Remarque p.140]). 
Example (5.2): An infinitesimal group action

Suppose $H$. is a real Hilbert space with scalar product $($,$) .$ Let $M=H$ and $G$ be $H$ with the additive group structure of $H$. Then $\mathrm{L}(\mathrm{G})=\mathrm{H}$ also. Define $\theta: \mathrm{L}(\mathrm{G}) \longrightarrow \mathrm{V}(\mathrm{M})$ by $\theta(\mathrm{Y})(\mathrm{X})=2(\mathrm{X}, \mathrm{Y}) \mathrm{X}-(\mathrm{X}, \mathrm{X}) \mathrm{Y}$. We show that $\theta$ is an infinitesimal action of $G$ on $M$.

(1) The map $\varepsilon:(\mathrm{Y}, \mathrm{X}) \longrightarrow \theta(\mathrm{Y})(\mathrm{X})$ is a vector bundle morphism from $L(G) \times M$ into $T(M): \varepsilon$ is obviously a morphism. Let Hom(H,H) denote the continuous linear maps from $H$ into $H$ and let $\delta_{X} \varepsilon \operatorname{Hom}(H, H)$ be the map $Y \longrightarrow \theta(Y)(X)$. We need that the map $X \longrightarrow \delta_{X}$ of $H$. into $\operatorname{Hom}(\mathrm{H}, \mathrm{H})$ is continuous, but this is the case since $():, \mathrm{H} \times \mathrm{H} \longrightarrow \mathrm{H}$ is continuous.

(2) $\theta$ is a Lie algebra homomorphism : $\theta$ is obviously linear. In order to prove that $\theta$ preserves brackets it suffices to show that $[\theta(Y), \theta(Z)]=0$ for any $Y$ and $Z$ in $L(G)$ since $L(G)=H$ is abelian. By definition

$$
[\theta(Y), \theta(Z)](X)=\left.D \theta(Z)\right|_{X}(\theta(Y)(X))-\left.D \theta(Y)\right|_{X}(\theta(Z)(X))
$$

A short calculation gives $\left.\mathrm{D} \theta(\mathrm{W})\right|_{\mathrm{X}}(\mathrm{H})=2((\mathrm{X}, \mathrm{Y}) \mathrm{H}+(\mathrm{H}, \mathrm{Y}) \mathrm{X}-(\mathrm{X}, \mathrm{H}))$ and substituting this into the above equation with $\mathrm{W}=\mathrm{Z}$ (and $\mathrm{Y}$ ) and $H=\theta(Y)(X)$ (and $\theta(Z)(X)$ ) makes the equation identically zero. Therefore $\theta$ preserves brackets. 
Suppose $\phi: D \longrightarrow M$ is a local action of $G$ on $M$. Define $\phi^{+}: L(G) \rightarrow V(M)$ by $\phi^{+}(v)(p)=T(\phi)\left(v(e), o_{p}\right)$ where $o_{p}$ is the zero vector in $T_{p}(M)$

Proposition (5.3) $\phi^{+}$is an infinitesimal action of $G$ on $M$.

Proof : Evaluation map of $\phi^{+}$is a vector bundle morphism :

We have the following sequence of maps

$$
\begin{aligned}
& \mathrm{L}(\mathrm{G}) \times \mathrm{M} \longrightarrow \mathrm{L}(\mathrm{G}) \times \mathrm{G} \times \mathrm{M} \stackrel{\beta \times \gamma}{\longrightarrow} \mathrm{TD} \stackrel{\mathrm{T}(\phi)}{\longrightarrow} \mathrm{TM} \\
& \quad(\mathrm{v}, \mathrm{p}) \longrightarrow(\mathrm{v}, \mathrm{e}, \mathrm{p}) \longrightarrow\left(\mathrm{v}(\mathrm{e}), \mathrm{o}_{\mathrm{p}}\right) \longrightarrow \mathrm{T}(\phi)\left(\mathrm{v}(\mathrm{e}), \mathrm{o}_{\mathrm{p}}\right)
\end{aligned}
$$

where $\beta$ is the trivializing vector bundle isomorphism $(v, g) \rightarrow v(g)$ of $L(G) \times G$ into $T(G)$ and $\gamma$ is the zero section. The fact that the evaluation map is a vector bundle morphism then follows from the fact that $B$ and $T(\phi)$ are.

$\phi^{+}$is a Lie algebra homomorphism $:$

$\phi^{+}$is obviously linear and therefore it remains to show that it preserves brackets. Let $p \in M$, suppose $(g, p) \varepsilon D$ and $\phi(g, p)=q$, then if $h \in D^{p^{-1}} \cap D^{q}$ we have $(h, q)=(h, \phi(g, p)),(h g, p)$ and $(\mathrm{g}, \mathrm{p}) \varepsilon \mathrm{D}$ which implies $\phi(\mathrm{h}, \mathrm{g})=\phi(\mathrm{h}, \phi(\mathrm{g}, \mathrm{p}))=\phi(\mathrm{hg}, \mathrm{p})$ by Def.(4.1) (2). This means $\phi^{q}=\phi^{p} \circ R(g)$ on the open set $D^{p} g^{-1} \cap D^{q}$ containing e which implies $T\left(\phi^{q}\right)=T\left(\phi^{p}\right) \circ T(R(g))$ on $T\left(D^{p} g^{-1} \cap D^{q}\right)$ and that $\mathrm{T}_{\mathrm{e}}(\mathrm{G}) \subset \mathrm{T}\left(\mathrm{D}^{\mathrm{p}} \mathrm{g}^{-1} \cap \mathrm{D}^{\mathrm{q}}\right)$. Then for $\mathrm{v} \varepsilon \mathrm{L}(\mathrm{G})$ we have 


$$
\text { 1.6. }
$$

$$
\begin{aligned}
\phi^{+}(\mathrm{v})\left(\phi^{\mathrm{p}}(\mathrm{g})\right)=\phi^{+}(\mathrm{v})(\mathrm{q}) & =\mathrm{T}(\phi)\left(\mathrm{v}(\mathrm{e}), \mathrm{o}_{\mathrm{q}}\right) \\
=\mathrm{T}\left(\phi^{\mathrm{q}}\right)(\mathrm{v}(\mathrm{e})) & =\mathrm{T}\left(\phi^{\mathrm{p}}\right) \circ \mathrm{T}(\mathrm{R}(\mathrm{g}))(\mathrm{v}(\mathrm{e}))=\mathrm{T}\left(\phi^{\mathrm{p}}\right)(\mathrm{v}(\mathrm{g}))
\end{aligned}
$$

which implies that $v$ and $\phi^{+}(v)$ are $\phi^{p}$-related vector fields. Then $\left[v, v^{\prime}\right]$ is $\phi^{p}$-related to $\left[\phi^{+}(v), \phi^{+}\left(v^{\prime}\right)\right]$ (Bourbaki. $[, 8.5 .6$ p.17]). Then $\phi^{+}$is a Lie algebra homornorphism for

$$
\begin{aligned}
\phi^{+}\left(\left[v, v^{\prime}\right]\right)(p) & =\phi^{+}\left(\left[v, v^{\prime}\right]\right)\left(\phi^{p}(e)\right) \\
& =T\left(\phi^{p}\right)\left(\left[v, v^{\prime}\right](e)\right) \\
& =\left[\phi^{+}(v), \phi^{+}\left(v^{\prime}\right)\right]\left(\phi^{p}(e)\right) \\
& =\left[\phi^{+}(v), \phi^{+}\left(v^{+}\right)\right]\left(p^{p}\right)
\end{aligned}
$$

where $p$ was an arbitrary point of $M$. This completes the proof.

$\phi^{+}$is called the infinitesimal generator of $\phi$. If an infinitesimal action $\theta$ of $G$ on $M$ is equal to $\phi^{+}$for some local action $\phi$ then $\theta$ is called generating.

Example (5.4) Let $\phi$ be the local action considered in Example (4.4). Let $X \in L(G)=\operatorname{Hom}(E, F)$. Then

$$
\begin{aligned}
\phi^{+}(X)(p) & =T(\phi)\left(X(0), o_{p}\right) \\
& =\left.\frac{d}{d t}\right|_{t=0} \phi(t X, p) \\
& =\left.\frac{d}{d t}\right|_{t=0} p \circ\left(t X \circ p+I_{F}\right)^{-1}=-p \circ X \circ p .
\end{aligned}
$$


$\$ 6$ The Infinitesima1 Graph

Let $\theta: L(G) \rightarrow V(M)$ be an infinitesimal left action and let $\mathrm{P}_{\mathrm{G}}: \mathrm{G} \times \mathrm{M} \longrightarrow \mathrm{G}$ and $\mathrm{P}_{\mathrm{M}}: \mathrm{G} \times \mathrm{M} \longrightarrow \mathrm{M}$ be the canonical projections. Define $f$ from $\mathrm{p}_{G} * \mathrm{~T}(\mathrm{G})$ into $\mathrm{p}_{\mathrm{M}}{ }^{* \mathrm{~T}(\mathrm{M})}$ by

$$
f_{(g, m)}(X(g))=\theta(X)(m)
$$

where $X(g)$ is the value of $X \in L(G)$ at $g$. (See $\S 2$ for definitions of $\mathrm{P}_{G}{ }^{* T}(G)$ and $\left.\mathrm{P}_{M}{ }^{*} \mathrm{~T}(\mathrm{M})\right)$. We have $\mathrm{f}\left(\mathrm{X}(\mathrm{g}), \mathrm{O}_{\mathrm{P}}\right)=\left(\mathrm{O}_{\mathrm{g}}, \theta(\mathrm{X})(\mathrm{p})\right)$ and $\mathrm{f}$ is a vector bundle morphism since the evaluation map $(X, p) \longrightarrow \theta(X)(p)$ was assumed to be a vector bundle morphism of $L(G) \times M$ into $T(M)$. Then prop.(2.1) implies that the graphs of the ff $(g, p)$, $\{(X(g), \theta(X)(p)): p \in M\}$, are the fibers of a subbundle $F^{f}$ of $\mathrm{T}(\mathrm{G}) \times \mathrm{T}(\mathrm{M}) . \quad \mathrm{F}^{\mathrm{f}}$ is called the infinitesimal graph of $\theta$.

Proposition (6.1) $\mathrm{F}^{\mathrm{f}}$ is an integrable subbundle of $\mathrm{T}(\mathrm{G}) \times \mathrm{T}(\mathrm{M})$.

Proof : Consider the family of sections $\left\{\xi_{X}\right\}_{X_{L L}(G)}$ of $F^{f}$ where $\xi_{X}(g, p)=(x(g), \theta(x)(p))$. Then

(1) by definition of $F^{\mathrm{f}}$ the set $\left\{\xi_{\mathrm{X}}(\mathrm{g}, \mathrm{p})\right\}_{\mathrm{X} \in \mathrm{L}(\mathrm{G})}$ is total in the fiber $F_{(g, p)}^{f}$ above $(g, p)$ in $F^{f}$, and

(2) if $(X, Y)$ is any pair of elements of $L(G)$ and if $(g, p) \varepsilon G \times M$ then

$$
\begin{aligned}
{\left[\xi_{\mathrm{X}}, \xi_{\mathrm{Y}}\right](\mathrm{g}, \mathrm{p}) } & =([\mathrm{X}, \mathrm{Y}](\mathrm{g}),[\theta(\mathrm{X}), \theta(\mathrm{Y})](\mathrm{p})) \\
& =([\mathrm{X}, \mathrm{Y}](\mathrm{g}), \theta([\mathrm{X}, \mathrm{Y}](\mathrm{p}))
\end{aligned}
$$


since $\theta$ is a Lie algebra homomorphism. This shows $\left[\xi_{X}, \xi_{Y}\right](g ; p) \varepsilon F^{f}(g, p)$ and the Theorem of Frobenius (1.5) implies $F^{f}$ is integrable.

By the definition of integrability there is a foliation $Y$. of $\mathrm{G} \times \mathrm{M}$ such that $\mathrm{T}(\mathrm{G} \times \mathrm{M}, \mathrm{Y})=\mathrm{F}^{\mathrm{f}}$.

Proposition (6.2) For: $g \in G$, let $\bar{R}(\mathrm{~g})$ be the morphism of $G \times M$ into itself given by $\bar{R}(g)(h, p)=(h g, p)$, then $\bar{R}(g)$ is also a morphism of $\mathrm{Y}$ into $\mathrm{Y}$ where $\mathrm{Y}$ is the foliation defined by any infinitesimal action $\theta$ of $G$ on $M$ :

Proof :

$$
\begin{aligned}
& T(\bar{R}(g))\left(T_{(h, p)}(G \times M, Y)\right) \\
= & T(\bar{R}(g))(\{(X(h), \theta(X)(p): X \varepsilon L(G)\}) \\
= & \{(X(h g), \theta(X)(p): X \varepsilon L(G)\} \\
= & T_{(h g, p)}(G \times M, Y)
\end{aligned}
$$

and prop. (1.4) implies that $\bar{R}(\mathrm{~g})$ is a morphism of $\mathrm{Y}$ into $\mathrm{Y}$.

Remark : Since $\bar{R}(\mathrm{~g})$ is a diffeomorphism it takes a maximal connected leaf of $Y$ diffeomorphically onto another maximal connected leaf of $Y$.

The next proposition explains the name "infinitesimal graph".

Proposition (6.3) If $\phi$ is any local left action with domain $D$ and infinitesimal generator $\phi^{+}$then the morphism $\phi^{p}: g \rightarrow \phi(g, p)$ of $D^{p}$ 
into $M$ is an integral (Def.(2.2)) of $f$ (where $f$ is defined as above with $\left.\theta=\phi^{+}\right)$. Also the graph of $\phi^{-p}$ is a leaf containing $(e, p)$ of the folliation $Y$ and the morphism $\pi_{G}: Y \longrightarrow G$ given by $\pi_{G}(g, p)=g$ is a local diffeomorphism at each point of $\mathrm{Y}$.

Proof : Let $X \in L(G)$. Then

$$
\begin{aligned}
\mathrm{T}_{\mathrm{g}}\left(\phi^{\mathrm{P}}\right)(\mathrm{X}(\mathrm{g})) & =\mathrm{T}_{\mathrm{g}}\left(\phi^{\mathrm{P}}\right) \circ \mathrm{T}_{\mathrm{e}}(\mathrm{R}(\mathrm{g}))(\mathrm{X}(\mathrm{e})) \\
& =\mathrm{T}_{\mathrm{e}}\left(\phi^{\mathrm{P}} \circ \mathrm{R}(\mathrm{g})\right)(\mathrm{X}(\mathrm{e})) \\
& =\mathrm{T}_{\mathrm{e}}\left(\phi^{\phi(\mathrm{g}, \mathrm{p})}\right)(\mathrm{X}(\mathrm{e})) \\
& =\phi^{+}(\mathrm{X})(\phi(\mathrm{g}, \mathrm{p}))
\end{aligned}
$$

Hence ${ }^{T}{ }_{g}{ }^{p}={ }^{f}\left(g, \phi^{P}(g)\right)$ and so $\phi^{p}$ is an integral of $f$. The fact that the graph of $\phi^{p}$ is a leaf containing (e, p) follows from prop. (2.5).

Let $(g, p)$ be any point in $Y$. Then $\pi_{G}$ is a local diffeomorphism at $(e, p)$ because $N_{p}=\left\{\left(h, \phi^{p}(h)\right): h \in D^{p}\right\}$ is an open neighbourhood of $(e, p)$ in $Y$ mapped diffeomorphically onto $D^{p}$ by $\pi_{G}$. Now $\bar{R}(g)\left(N_{p}\right)$ is an open neighbourhood of $(g, p)$ in $Y$ by the remark after prop. (6.2) and $\pi_{G}$ is a local diffeomorphism on $\bar{R}(g)\left(N_{p}\right)$ which completes the proof.

We now show that two local actions with the same infinitesimal generator coincide in a neighbourhood of $\{e\} \times M$. We need a lemma. 
Lemma (6.4). If an infinitesimal action $\theta$ of $G$ on $M$ is generating then the foliation $Y$ defined by the infinitesimal graph of $\theta$ is a Hausdorff manifold.

Proof : See Palais [7, Theorem VIII, p.44].

Note : Palais' definition of leaf differs slightly from ours:

Let $\phi$ and $\psi$ be local actions of $G$ on $M$ with domains $D_{\phi}$ and $\mathrm{D}_{\psi}$ respectively. Let $\mathrm{D}_{\mathrm{p}}$ be the connected component of $\mathrm{e}$ in $\mathrm{D}_{\phi}^{\mathrm{p}} \cdot \cap \mathrm{D}_{\psi}^{\mathrm{p}}$, then $\mathrm{D}=\bigcup_{\mathrm{p} \varepsilon \mathrm{M}} \cdot \mathrm{D}_{\mathrm{p}} \times\{\mathrm{p}\}$ is an open neighbourhood of $\{\mathrm{e}\} \times \mathrm{M}$ in $G \times M$ (Palais [7, Theorem 1, p.32]).

Uniqueness Theorem (6.5) If $\phi$ and $\psi$ have the same infinitesimal generator $\theta$ then $\phi$ and $\psi$ coincide on $D$.

Proof : By prop. (6.3) both $\phi^{\mathrm{p}}$ and $\psi^{\mathrm{p}}$ are integrals of $\mathrm{f}$ (where $\mathrm{f}$ is defined as in prop.(6.3)). Let $A \subset D_{p}$ be the set of points on which $\phi^{\mathrm{P}}$ and $\psi^{\mathrm{P}}$ agree. $\mathrm{A}$ is nonempty since $\phi^{\mathrm{p}}(\mathrm{e})=\psi^{\mathrm{P}}(\mathrm{e})=\mathrm{p}$. Prop. (2.3) implies that $A$ is open. Let $Y$ as usual be the foliation defined by the infinitesimal graph of $\theta$. A is closed in $D_{p}$ since $A=\Phi^{-1}(\Delta)$ where $\Phi$ is the morphism from $D_{p}$ into $Y \times Y$ given by $\Phi(g)=\left(\phi^{p}(g), \psi^{p}(g)\right)$ and $\Delta$ is the diagonal in $\mathrm{Y} \times \mathrm{Y}$ which is a closed set since $\mathrm{Y}$ is Hausdorff (Lemma (6.4)). Then $A=D_{p}$ since $D_{p}$ is connected. 


\section{§7. Existence Theorem}

We now give necessary and sufficient conditions on $M$ for an infinitesimal action of $G$ on $M$ to be generating.

Theorem (7.1) A necessary and sufficient condition that an infinitesimal action $\theta$ of $G$ on $M$ is generating is that the foliation defined by the infinitesimal graph of $\theta$ is a Hausdorff manifold.

Proof : This theorem is proven in Palais [7, pp.52-58] for finite dimensional M. The same proof works in infinite dimensions. A weaker theorem giving sufficient (but not necessary) conditions for $\theta$ to be generating is proven in Bourbakj $[-2, \text { Cor } .1,184]^{\circ}$.

Example (7.2) : Local action generated by an infinitesimal action

Consider the infinitesimal action defined in Example (5.2). Keeping the same notation, let $\exp : L(G) \longrightarrow G$ be the exponential map, then $\exp =i d$. If $\mathrm{X} \in \mathrm{V}(\mathrm{M})$, let $\delta_{\mathrm{X}, t}$ denote the local one-parameter group defined by $X$. Now if $\phi$ is a local action of $G$ on $M$ such that $\phi^{+}=\theta$ then $\phi(t Y, p)=\phi(\exp t Y, p)=\delta_{\phi^{+}(Y), t}(p)$ by the uniqueness theorem for differential equation and definition of $\phi^{+}$. Therefore in order to find the local action $\phi$ corresponding to $\theta$ we must find the local one-parameter group corresponding to $\theta(\mathrm{Y})$. To shorten notation we will denote $(p, p)$ by $p^{2}$ and $(p, p)(p, p)$ by $p^{4}$ for $p \varepsilon H$. Now we have 


$$
\delta_{\theta(Y), t}(p)=\frac{p-t(p, p) Y}{1-2 t(p, Y)+t^{2}(p, p)(Y, Y)}
$$

for

$$
\begin{aligned}
& \text { (1) } \delta_{\theta(Y), 0}(\mathrm{p})=\mathrm{p} \\
& \text { (2) } \frac{\mathrm{d}}{\mathrm{dt}} \delta_{\theta(Y), t}(\mathrm{p})=\theta(\mathrm{Y})\left(\delta_{\theta(Y), t}(\mathrm{p})\right) .
\end{aligned}
$$

Proof of (2) : We have

$$
\begin{aligned}
\frac{d}{d t} \delta_{\theta(Y), t}(p) & =\left\{\frac{-p^{2}}{1-2 t(p, Y)+t^{2} p^{2} \dot{Y}^{2}}\right\} Y \\
& +\frac{\left\{2(p, Y)-2 t p^{2} Y^{2}\right\}}{\left\{1-2 t(\dot{p}, Y)+t^{2} p^{2} Y^{2}\right\}^{2}}\left\{p-t p^{2} Y\right\}
\end{aligned}
$$

and

$$
\begin{aligned}
& \theta(\mathrm{Y})\left(\delta_{\theta(\mathrm{Y}), t}(\mathrm{p})\right)=2\left\{\delta_{\theta(\mathrm{Y}), t}(\mathrm{p})\right\}\left(\delta_{\theta(\mathrm{Y}), t}(\mathrm{p}), \mathrm{Y}\right) \\
& -\left(\delta_{\theta(Y), t}(\mathrm{P}), \delta_{\theta(Y), t}(\mathrm{p})\right) Y \\
& =2\left\{\frac{\mathrm{p}-t \mathrm{p}^{2} Y}{1-2 t(\mathrm{p}, \mathrm{Y})+\mathrm{t}^{2} \mathrm{p}^{2} \mathrm{Y}^{2}}\right\}\left(\frac{\mathrm{p}-t \mathrm{p}^{2} \mathrm{Y}}{1-2 t(\mathrm{p}, \mathrm{Y})+\mathrm{t}^{2} \mathrm{p}^{2} \mathrm{Y}^{2}}, \mathrm{Y}\right) \\
& -\frac{1}{\left\{1-2 t(p, Y)+t^{2} p^{2} Y^{2}\right\}^{2}}\left(p-t p^{2} Y, p-t p^{2} Y\right) Y \\
& =2\left\{p-t p^{2} Y\right\}\left\{\frac{\left\{(p, Y)-t p^{2} Y^{2}\right\}}{\left\{1-2 t(p, Y)+t^{2} p^{2} Y^{2}\right\}^{2}}\right\} \\
& +\frac{\left\{-p^{2}\right\}}{\left\{1-2 t(p, Y)+t^{2} p^{2} Y^{2}\right\}} Y
\end{aligned}
$$


Comparing these two equations we see that (2) is true. Let

$$
D=\{(Y, p) \varepsilon G \times M \mid 1-2(p, Y)+(p, p)(Y, Y) \neq 0\} .
$$

$D$ is open and contains $\{0\} \times M$. Finally define $\phi: D \rightarrow M$ by

$$
\phi(Y, p)=\frac{p-(p, p) Y}{1-2(p, Y)+(p, p)(Y, Y)} .
$$

We complete this chapter with a discussion of a special type of infinitesimal action.

\section{$\$ 8$ Uniform Infinitesimal Actions}

Let $\theta: L(G) \longrightarrow V(M)$ be an infinitesimal left group action and $\Sigma_{p}$ be the maximal connected leaf through $(e, p)$ of the foliation $Y$ defined by $\theta \cdot \pi_{G}: Y \longrightarrow M$ is the morphism given by $\pi_{G}(g, p)=g$.

Definition (8.1) $\theta$ is called a uniform infinitesimal (left) action of $G$ on $M$ if there exists a connected neighbourhood $V$ of $e$ in $G$ such that for each $p \in M$ the connected component containing $(e, p)$ in $\Sigma_{p} \cap \pi_{G}^{-1}(V)$ is mapped one-to-one onto $V$ by $\pi_{G} \cdot V$ is called a uniform neighbourhood for $\theta$.

Theorem (8.2) Each maximal connected leaf $\Sigma$ of $Y$ is a covering space for $G$ with covering map $\pi \cdot=\left.\pi_{G}\right|_{\Sigma}$ if and only if $\theta$ is uniform. 
Proof : Suppose $\theta$ is uniform. Let $V$ be a uniform neighbourhood. We have to show that for each $g \varepsilon G$ there exists an open neighbourhood $W$ such that $\pi^{-1}(W)$ is a disjoint union of open sets in $\Sigma$, each of which is mapped diffeomorphically onto $W$ by $\pi$. We first show that $\pi(\Sigma)=G$ : Let $(\mathrm{g}, \mathrm{p}) \varepsilon \Sigma$, then by prop. $(6.2), \bar{R}\left(\mathrm{~g}^{-1}\right)(\Sigma)=\Sigma_{\mathrm{p}}$ since. $\bar{R}\left(g^{-1}\right)(g, p)=(e, p)$ and $\pi(\Sigma)=\pi \circ \bar{R}(g)\left(\Sigma_{p}\right)=R(g) \circ \pi_{G}\left(\Sigma_{p}\right)$. So if $\pi_{G}\left(\Sigma_{p}\right)=G$ then $\pi(\Sigma)=G$ also. This will be proven by showing that for every positive integer $n ; v^{n} \subset \pi_{G}\left(\Sigma_{p}\right)$, then $\pi_{G}\left(\Sigma_{p}\right)$ will equal $G$ since any neighbourhood of $e$ in a connected group generates the group. Since, $V$ is a uniform neighbourhood for $\theta$ this is true for $n=1$. Assume now that $v^{n-1} \subset \pi_{G}\left(\Sigma_{p}\right)$, we will show that $v^{n} \subset \cdot \pi_{G}\left(\Sigma_{p}\right)$ also. Let $g$ be any point of $\cdot v^{n-1}$ then by the induction hypothesis there exists $\mathrm{q} \cdot \varepsilon \mathrm{M}$ such that $(\mathrm{g}, \mathrm{q}) \varepsilon \Sigma_{\mathrm{p}}$. By prop. (6.2), $\bar{R}\left(\mathrm{~g}^{-1}\right)\left(\Sigma_{\mathrm{p}}\right)=\Sigma_{\mathrm{q}}$. Now $\mathrm{V} \subset \pi_{G}\left(\Sigma_{\mathrm{q}}\right)$ since $\mathrm{V}$ is uniform and so $\left.\mathrm{V} \subset \pi_{\mathrm{G}}{ }^{\circ} \bar{R}^{-1}\right)\left(\Sigma_{\mathrm{p}}\right)=\mathrm{R}\left(\mathrm{g}^{-1}\right) \circ \pi_{\mathrm{G}}\left(\Sigma_{\mathrm{p}}\right)$. This means $g V \subset \pi_{G}\left(\Sigma_{p}\right)$ for each $g \varepsilon v^{n-1}$, i.e. $v^{n} \subset \pi_{G}\left(\Sigma_{p}\right)$.

Now let $g$ be any point of $G$. Let $U$ be a symmetric connected neighbourhood of $e$ in $G$ such that $U^{2} \subset v$, then $W=U g$ is a neighbourhood of $g$. We will show that $\pi^{-1}(W)$ is a disjoint union of open sets in $\Sigma$, each of which is mapped diffeomorphically onto $W$ by $\pi$. Since $\pi(\Sigma)=G$ we have $\pi^{-1}(W)$ is nonempty. Let $C$ be any component in $\Sigma$ of $\pi^{-1}(W)=\pi^{-1}(U g)$. If $(h, s)$ is any point of $c$ then h $\varepsilon$ Ug which means $g h^{-1} \varepsilon U^{-1}=U$ and $\mathrm{Ugh}^{-1} \subset \mathrm{UU} \subset \mathrm{V}$. This implies that $\mathrm{Ugh}^{-1}$ is a uniform neighbourhood for $\theta$ since $V$ was. Prop. (6.2) gives $\bar{R}\left(h^{-1}\right)(\Sigma)=\Sigma_{s}$ since $\bar{R}\left(h^{-1}\right)(h ; s)=(e, s)$ and $\bar{R}\left(h^{-1}\right)(c)$ is the 
component of $(\mathrm{e} ; \mathrm{s})$ in $\Sigma_{\mathrm{S}} \cap \pi_{\mathrm{G}}^{-1}\left(\mathrm{Ugh}^{-1}\right)$. But $\pi_{G}$ maps the component of $(\mathrm{e}, \mathrm{s})$ in $\Sigma_{\mathrm{s}} n \pi_{\mathrm{G}}^{-1}\left(\mathrm{Ugh}{ }^{-1}\right)$ diffeomorphically onto $\mathrm{Ugh}^{-1}$ since $\mathrm{Ugh}^{-1}$ is a uniform neighbourhood which means $\pi$ maps $C$ diffeomorphically onto $\bar{R}(\mathrm{~h})\left(\mathrm{Ugh}^{-1}\right)=U g$ and therefore the pair $(\Sigma, \pi)$ is a covering space for $G$.

Conversely suppose $\left.\pi_{G}\right|_{\Sigma_{q}}: \Sigma_{q} \rightarrow G$ is a covering map. Let $V$ be any simply connected open neighbourhood of $e$. Then the component containing $(e, q)$ in $\pi_{G}^{-1}(v) \cap \Sigma_{q}$ is a covering space for $V$ and therefore must be mapped diffeomorphically onto $V$.

We will need the following theorem in Chapter 3 .

Theorem (8.3) If $G$ is simply connected and $M$ is a Hausdorff manifold then a uniform infinitesimal left action $\theta: L(G) \rightarrow V(M)$ generates a global action of $G$ on $M$.

Proof : By the above theorem each leaf $\Sigma$ is a covering space for $G$ and. since $G$ is simply connected $\left.\pi_{G}\right|_{\Sigma}: \Sigma \longrightarrow G$ is a diffeomorphism. For $p \varepsilon M$ denote this diffeomorphism of $\Sigma_{p}$ onto $G$ by $\pi_{G}^{P}$. As usual denote by $f$, the vector bundle morphism from $p_{G}{ }^{* T}(G)$ into $p_{M}{ }^{* T}(M)$ induced by $\theta$, and by $Y$ the foliation of $G \times M$ defined by the integrable subbundle $F^{f}$ Define $\phi^{p}: G \rightarrow M$ to be $\phi^{p}(g)=\pi_{M}{ }^{\circ}\left(\pi_{G}^{p}\right)^{-1}(g)$ where $\pi_{M}: Y \rightarrow M$ is $\pi_{M}(g, m)=m$. Finally define $\phi: G \times M \longrightarrow M$ to be $\phi(g, p)=\phi^{p}(g)$. We will show that $\phi$ is a global group action with infinitesimal generator 
$\theta$. Let $v \in L(G)$. Note that each ${ }_{\phi}^{p}$ is an integral for $f$ since

$$
\begin{aligned}
T\left(\phi^{p}\right)(v(g)) & =T\left(\pi_{M}\right) \circ T\left(\pi_{G}^{p^{p}}\right)^{-1}(v(g)) \\
& =T\left(\pi_{M}\right)\left(v(g), f\left(g,\left(\pi_{G}^{p}\right)^{-1}(g)\right)^{(v \cdot(g)))}\right. \\
& =f\left(g, \phi^{p}(g)\right)^{(v(g))} \text { as }\left(\pi_{G}^{p}\right)^{-1}(g)=\phi^{p}(g) .
\end{aligned}
$$

$\phi$ is a global action :

(1) $\phi(e, p)=\pi_{M} \circ\left(\pi_{G}^{p}\right)^{-1}(e)=\pi_{M}(e, p)=p$ since $(e, p)$ is the unique point in $\Sigma_{p}$ with first component equal to $e$.

(2). Show $\phi(\mathrm{g}, \phi(\mathrm{h}, \mathrm{p}))=\phi(\mathrm{gh}, \mathrm{p})$ for all $\dot{\mathrm{g}}, \mathrm{h} \varepsilon \mathrm{G}$ and $\mathrm{p} \varepsilon \mathrm{M}$. Define $\psi_{1}(g)=\phi(g, \phi(h, p))$ and $\psi_{2}(g)=\phi(g h, p)$. By the definition of $\phi$, the graph of $\psi_{1}$ is $\Sigma_{\phi(h, p)}$ and the graph of $\psi_{2}$ is $\bar{R}\left(h^{-1}\right)\left(\Sigma_{p}\right)=\Sigma_{\phi(h, p)} \quad$ since $: \bar{R}\left(h^{-1}\right)(h, \phi(h, p))=(e, \phi(h, p))$. Then since $\pi_{G}^{\phi(h, p)}$ is one-to-one on $\Sigma_{\phi(h, p)}$ we have $\phi(g, \phi(h, p))=\phi(g h, p)$. We now show that $\phi: G \times M \longrightarrow M$ is a morphism. For $p \varepsilon M$, define

$$
\begin{aligned}
\mathrm{A}^{\mathrm{P}}=\{\mathrm{g} \varepsilon \mathrm{G}: & \text { there exists some open neighbourhood } \mathrm{U} \\
& \text { of } \mathrm{g} \text { and some open neighbourhood } \mathrm{V} \text { of } \\
\mathrm{p} \text { such that } \phi \text { is a morphism on } \mathrm{U} \times \mathrm{V}\} &
\end{aligned}
$$


(a) $A^{p}$ contains $e$ and therefore $A^{P} \neq \emptyset$.

Let $\rho: M \longrightarrow M$ be the identity. By prop. (2.6) there exists a connected open neighbourhood $U \times V$ of $(e, p)$ in $G \times M$ and a morphism $\psi: \mathrm{U} \times \mathrm{V} \longrightarrow \mathrm{M}$. such that for all $\mathrm{m} \varepsilon \mathrm{V}$ the morphism. $\psi^{\mathrm{m}}: \mathrm{g} \longrightarrow \psi(\mathrm{g}, \mathrm{m})$ is an integral for $f$ with $\psi^{\mathrm{n}}(\mathrm{e})=\rho(\mathrm{m})=\mathrm{m} \cdot \phi^{\mathrm{m}}$ is also an integral for $\mathrm{f}$ on $\mathrm{U} \times \mathrm{V}$ with $\phi^{\mathrm{m}}(\mathrm{e})=\psi^{\mathrm{m}}(\mathrm{e})$. Since $\mathrm{M}$ is Hausdorff and $\mathrm{U}$ is connected it follows, just as in the proof of theorem (6.4) using the uniqueness of integrals, that $\phi^{\mathrm{m}}=\psi^{\mathrm{m}}$ on $\mathrm{U}$; i.e. $\phi=\psi$ on $\mathrm{U} \times \mathrm{V}$ and $\mathrm{A}^{\mathrm{p}}$. contains $\mathrm{e}$.

(b) $\underline{A}^{P}$ is open in $G$ by definition.

(c) $\mathrm{A}^{\mathrm{P}}$ is closed in $\mathrm{G}$.

Let $g \in \overline{A^{\mathrm{p}}}$, by (a) above there exists a connected neighbourhood $\mathrm{U} \times \mathrm{V}$ of $(\mathrm{e}, \phi(g, p))$ such that $\phi$ is a morphism on $\mathrm{U} \times \mathrm{V}$. We denote $\phi$ by $\beta$ on $U \times V$ to emphasize that it is a morphism. Furthermore we assume $\mathrm{U}=\mathrm{U}^{-1}$. Since $\mathrm{h} \rightarrow \phi(\mathrm{h}, \mathrm{p})$ is an integral, and so in particular continuous, there exists a neighbourhood $N$ of $g$ such that $\phi(N, p) \subset v$. Let $\mathrm{h} \in \mathrm{N} \cap \mathrm{Ug} \cap \mathrm{A}^{\mathrm{p}} ; \mathrm{h}$ exists since $\mathrm{g} \varepsilon \overline{\mathrm{A}^{\mathrm{p}}}$ and $\mathrm{N} \cap \mathrm{Ug}$ is a neighbourhood of $\mathrm{g}$. By the definition of $\mathrm{A}^{\mathrm{p}}$ there exists a connected neighbourhood $U_{1} \times v_{1}$ of $(h, p)$ on which $\phi$ is a morphism and since $\phi(h, p) \varepsilon V$ we can assume (shrinking if necessary) that $\phi\left(h, v_{1}\right) \subset v \cdot$ Define $\gamma:$ Uh $\times v_{1} \rightarrow M$ by $\gamma(k, m)=\beta\left(k^{-1}, \phi(h, m)\right) . \gamma$ is a morphism on Uh $\times V_{1}$ since it is a composition of morphisms; $\gamma=\beta \circ\left(R\left(h^{-1}\right) \times \phi^{h}\right)$. Now for a $\varepsilon v_{1}$, we have the morphism $\dot{\gamma}^{m}: U_{1} \rightarrow M$ given by 
$\gamma^{m}(k)=\gamma(k, m)$ with $\gamma(h, m)=\phi(h, m)$. We will show that $\gamma^{m}$ is an integral for $f$ and then (as in the proof of (a)) since $\phi^{m}$ is also an integral with the same value at $h, \phi^{\mathrm{m}}$ will equal $\gamma^{\mathrm{m}}$ and $\phi$ will be a morphism on $\mathrm{Uh} \times \mathrm{V}_{1}$. Let $\beta^{\mathrm{m}}: \mathrm{U} \longrightarrow \mathrm{M}$ be the morphism defined by $\beta^{m}(k)=\beta(k, m)$. Since $\beta=\phi$ on $U \cdot x \cdot V$ we have $\beta^{m}=\phi^{m}$ on $U$. Let $X \in L(G)$, to show that $\gamma$ is an integral", we need that

$$
T_{g}\left(\gamma^{m}\right)(X(g))=f_{\left(g, \gamma^{m}(g)\right)}(X(g))=\theta(X)\left(\gamma^{m}(g)\right)
$$

We have

$$
\begin{aligned}
& \mathrm{T}_{\mathrm{g}}\left(\gamma^{\mathrm{m}}\right)(\mathrm{X}(\mathrm{g}))=\mathrm{T}_{\mathrm{g}}\left(\beta^{\phi(h ; \mathrm{m})} \circ \mathrm{R}\left(\mathrm{h}^{-1}\right)\right)(\mathrm{X}(\mathrm{g})) \text { (by def. of } \gamma \text { ) }
\end{aligned}
$$

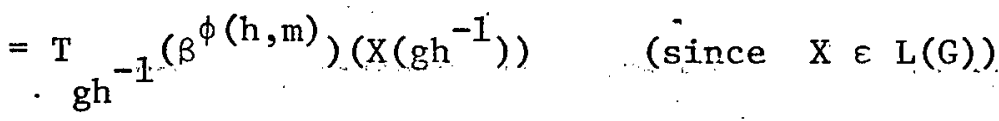

$$
\begin{aligned}
& =\theta(X)\left(\beta^{\phi(h, m)}\left(g h^{-1}\right)\right)
\end{aligned}
$$

(since $\beta^{\phi(h, m)}=\phi^{\phi(h, m)}$ is an integral of $f$ )

$$
\begin{aligned}
& =\theta(X)\left(\beta^{\phi(h, m)} \cdot R\left(h^{-1}\right)(g)\right) \\
& =\theta(X)\left(\gamma^{m}(g)\right)
\end{aligned}
$$

and so $\gamma^{\mathrm{m}}$ is an integral of $\mathrm{f}$. Therefore $\phi$ is a morphism on $\mathrm{Uh} \times \mathrm{V}_{1}$ and $h \varepsilon U g$ implies $g \in U^{-1} h=U h$, i.e. $(g, p) \varepsilon U h \times V_{1}$ and $g \varepsilon A^{p}$ showing that $\cdot \overrightarrow{A^{P}}=\overline{A^{P}}$. Since $G$ i.s connected (a), (b) and (c) imply that $A^{P}=G$. As $p$ was arbitrary $\phi$ is a morphism on $G \times M$.

It remains to show that $\theta$ is the infinitesimal generator of $\phi$. 
Let $X \in L(G)$, then

$$
\begin{aligned}
\mathrm{T}_{\mathrm{e}} \phi^{\mathrm{p}}(\mathrm{X}(\mathrm{e})) & =\mathrm{f}{ }_{\left(\mathrm{e}, \phi^{\mathrm{p}}(\mathrm{e})\right)^{(\mathrm{X}(\mathrm{e})} \quad \text { (since } \phi^{\mathrm{p}} \text { is an }}^{\text {integral for } \mathrm{f})} \\
& =\theta(\mathrm{X})\left(\phi^{\mathrm{p}}(\mathrm{e})\right)=\theta(\mathrm{X})(\mathrm{p})
\end{aligned}
$$

showing that $\theta$ is the infinitesimal generator: of: $\phi$ and completing the proof of the theorem.

The proof that $\phi$ is a morphism is essentially the same as the proof showing that the flow of a vector field is a morphism. (Cf. Lang $[5, \mathrm{p} .80])$.

Proposition (8.4) Let $\phi: G \times M \rightarrow M$ be a global left action of $G$ on a Hausdorff manifold $M$. Let $X \in L(G)$ and $\left\{\delta_{t}\right\}$ be the one-parameter group corresponding to $\phi^{+}(\mathrm{X})$. Then

$$
\delta_{t}(p)=\phi\left(\exp _{G} t X, p\right) \quad \text { for all } p \varepsilon M
$$

Proof : $\quad \phi\left(\exp _{G} 0 \cdot x, p\right)=\phi(e, p)=p$

and

$$
\begin{aligned}
\frac{\mathrm{d}}{\mathrm{dt}} \phi\left(\exp _{\mathrm{G}} \mathrm{tX}, \mathrm{p}\right)= & \left.\frac{\mathrm{d}}{\mathrm{ds}}\right|_{\mathrm{s}=0} \phi\left(\exp _{\mathrm{G}}(\mathrm{s}+\mathrm{t}) \mathrm{x}, \mathrm{p}\right) \\
= & \left.\frac{\mathrm{d}}{\mathrm{ds}}\right|_{\mathrm{S}=0} \phi\left(\exp _{\mathrm{G}} \mathrm{sX}, \phi\left(\exp _{\mathrm{G}} \mathrm{tX}, \mathrm{p}\right)\right) \\
& \cdot{ }^{+}(\mathrm{x})\left(\phi\left(\exp _{\mathrm{G}} \mathrm{tX}, \mathrm{p}\right)\right) \text { by definition of } \phi^{+} .
\end{aligned}
$$

The result then follows from the uniqueness theorem for differential equations. 
Chapter 3

\section{Connected Lie. Transformation Groups}

Let $D(M)$ be the group of diffeomorphisms of the manifold $M$. A Lie group $G$ is called a Eie transformation group of $M$ if the" underlying group of $G$ is a subgroup of $D(M)$ and if the map $(g, p) \rightarrow g(p)$ of $G \times M$ into $M$ is a morphism. of course one could give $G$ the discrete topology and this would automatically be true. A nontrivial example is the group $I(M)$ of isometries of a finite dimensional Riemannian manifold, which is a Lie transformation group with respect to the compact open topology. Further examples of lie transformation groups can be found in $\mathrm{H}$. Chu and S. Kobayashi [3]. The main result of this chapter is to show that there is a one-to-one correspondence between connected Lie transformation groups of $M$ and certain subalgebras of the Lie algebra of vector fields $V(M)$ where $M$ is a Hausdorff manifold. In this chapter $M$ will always denote a Hausdorff manifold.

\$9 The Image of the Infinitesimal Generator of a Lie Transformation Group

Let $G$ be a Lie transformation group of $M$. Then there is a global action $\phi$ of $G$ on $M$ with infinitesimal generator $\phi^{+}: L(G) \longrightarrow V(M)$. We now examine the image of $\phi^{+}$in $V(M)$. Let $\exp : \mathrm{L}(\mathrm{G}) \longrightarrow G$ be the exponential map. 
Proposition (9.1) The image $\phi^{+}(L(G))$ consists of complete vector fields and the one-parameter group corresponding to $\phi^{+}(X)$ is exp tX.

Proof : exp tx is a one-parameter group and

$$
\left.\frac{d}{d t}\right|_{t=0} \exp t X(p)=\phi^{+}(X)(p)
$$

The result then follows from the uniqueness theorem for differential equations.

Proposition $(9.2) \phi^{+}$is injective.

Proof : If $\phi^{+}(X)=0$ then

$$
\begin{aligned}
\left.\frac{d}{d t}\right|_{t=s} \exp t X(p) & =\left.\frac{d}{d t}\right|_{t=0} \exp ((s+t)(X)(p) \\
& =\left.\frac{d}{d t}\right|_{t=0} \exp t X(\exp s X(p)) \\
& =\phi^{+}(X)(\exp \operatorname{sX}(p)) \\
& =0 \quad \text { for all } p \in M
\end{aligned}
$$

This means $\exp t X(p)=p$ for all $t \varepsilon R$ and all $p \varepsilon M, i . e$ exp $t X=i d_{M}$ which implies that $\mathrm{x}=0$ since $\exp$ has a radius of injectivity at 0 in $L(G)$.

Proposition (9.3) $\phi^{+}(\mathrm{L}(\mathrm{G}))$ possesses a Banach Lie algebra structure such that the evaluation map $(Y, p) \rightarrow Y(p)$ is a vector bundle morphism from 
the trivial vector bundle $\phi^{+}(L(G)) \times M$ into $T(M)$ and

$\phi^{+}: L(G) \rightarrow \phi^{+}(L(G))$ is a Banach Lie algebra isomorphism. Furthermore, this Banach space structure is necessarily unique.

Proof : By prop. (9.2) $\phi^{+}: L(G) \longrightarrow \phi^{+}(L(G))$ is a Lie algebra isomorphism and hence induces a Banach Lie algebra structure on $\phi^{+}(\mathrm{L}(\mathrm{G}))$ making $\phi^{+}$a Banach Lie algebra isomorphism: Define $\beta: \phi^{+}(L(G)) \times M \longrightarrow L(G) \times M$ by $\beta(Y, p)=\left(\left(\phi^{+}\right)^{-1}(Y), p\right)$, then $\beta$ is easily seen to be a vector bundle morphism. Now prop. (5.3) gives that the map $\alpha:(X, p) \rightarrow \phi^{+}(X)(p)$ of $L(G) \times M \longrightarrow T(M)$ is a vector bundle morphism. The evaluation map $\phi^{+}(\mathrm{L}(\mathrm{G})) \times \mathrm{M} \longrightarrow \mathrm{T}(\mathrm{M})$ is equal to $\alpha \circ \beta$ and therefore is a vector bundle morphism. The uniqueness of the Banach space structure comes from the following proposition.

Proposition (9.4) Let $E$ be a vector bundle over $M$ and let $V$ be a vector space of sections of $\mathrm{E}$. If $\mathrm{V}$ admits two Banach space structures such that the evaluation map $(X, p) \rightarrow X(p)$ of $V \times M$ into $E$ is continuous with respect to both then the identity map from $V$ into $V$ is a homeomorphism, i.e. the two norms are equivalent.

Proof : Let $V_{1}$ and $V_{2}$ denote $V$ with respect to the two topologies and let $e_{i}: V_{i} \times M \rightarrow E \quad(i=1,2)$ be the evaluation maps. By the closed graph theorem, in order to show that id : $V_{1} \rightarrow V_{2}$ is continuous, it is enough to show that the diagonal is closed in $v_{1} \times v_{2} \cdot$ Let $\left\{\left(x_{n}^{1}, x_{n}^{2}\right)\right\}$, be a Cauchy sequence in the diagonal of $v_{1} \times v_{2}$, i.e. $\mathrm{x}_{\mathrm{n}}^{1} \varepsilon \mathrm{V}_{1}, \mathrm{x}_{\mathrm{n}}^{2} \varepsilon \mathrm{V}_{2}$ and $\mathrm{x}_{\mathrm{n}}^{1}=\mathrm{x}_{\mathrm{n}}^{2}$. Since $\mathrm{V}_{1} \times \mathrm{V}_{2}$. is complete there exists 
a limit point (X, Y) of this sequence; but for all $\mathrm{p} \varepsilon \mathrm{M}$ we have

$$
\begin{aligned}
X(p)=\lim _{n \rightarrow \infty} e_{1}\left(x_{n}^{1}, p\right) & =\lim _{n \rightarrow \infty} x_{n}^{1}(p) \\
& =\lim _{n \rightarrow \infty} x_{n}^{2}(p) \\
& =\lim _{n \rightarrow \infty} e_{2}\left(X_{n}^{2}, p\right)=Y(p)
\end{aligned}
$$

Therefore $\mathrm{X}=\mathrm{Y}$ and the diagonal is closed in $\mathrm{V}_{1} \times \mathrm{V}_{2}$. Interchanging $V_{1}$ and $V_{2}$ above gives that id $: V_{2} \rightarrow V_{1}$ is continuous also and id is a homeomorphism.

If $\mathrm{Y}$ is a complete vector field then denote by Exp tY, the one-parameter group generated by $Y$. Let $\phi: G \times M \rightarrow \bar{M}$ be the global action of a Lie transformation group G. Prop.(9.1) gives that $\exp _{G}(X)=\operatorname{Exp}\left(\phi^{+}(X)\right)$ and this implies the following result.

Proposition (9.5) Exp is injective on a neighbourhood of 0 in $\phi^{+}(L(G))$ in the topology induced on $\phi^{+}(L(G))$ by $\phi^{+}$.

$\$ 10$ Banach Lie Algebras of Complete Vector Fields

We now consider when a Lie subalgebra $L$ of $V(M)$, is the image of the infinitesimal generator of a connected Lie transformation group. In view of propositions (9.1), (9.3), and (9.5) we only consider Lie subalgebras $L$ of $V(M)$ which satisfy the following conditions

(A). L consists of complete vector fields; 
(B) I has a Banach Lie algebra structure, (necessarily unique by Prop. (9.4)) such that ;

(BI) the evaluation map ev $:(X, p) \rightarrow X(p)$ is a vector bundle morphism from the trivial bundle $L \times M$ into $T(M)$.

(B2) there exists an open ball $B_{r}(0)$ of. radius $r$ at. 0 such that $\operatorname{Exp}: L \rightarrow D(M)$ is injective on $B_{r}(0)$.

Proposition (10.1) If $I$ is finite dimensional and satisfies (A) then condition (B) is true also.

Proof : Since $\mathrm{L}$ is finite dimensiional it has a natural Banach space structure. A proof of (B1) is in Bourbaki [2, Remarque p.140] and a proof of "(B2) is in Loos $[6, p .182]$.

The rest of this section will be devoted to proving the following theorem.

Theorem (10.2) If $M$ is a Hausdorff manifold and $L$ is a Lie subalgebra of $V(M)$ satisfying conditions (A) and (B) then there exists a unique connected Lie transformation group $G$ with natural global action $\phi: G \times M \rightarrow M$ such that $\phi^{+}$is a Banach Lie algebra isomorphism of $\mathrm{L}(\mathrm{G})$ onto L.

Remark : Palais [7] first proved this theorem in the case where $L$ and $M$ are finite dimensional. Using a different method, Loos [6] extended this result to the case where $L$ is finite dimensional and $M$ is a (not 
necessarily Hausforff) Banach mainfold. The proof given here is similar to Palais' .

In order to prove Theorem (10.2) we need the following theorem, which is of interest in itself.

Theorem (10.3). If $M$ is a Hausdorff manifold and $L$ is a Lie subalgebra of $V(M)$ satisfying condition (A) and admitting a Banach Lie algebra structure such that (B1) is true (but not necessarily (B2)) then there exists a simply connected Lie group $\tilde{G}$ with $L(\tilde{G})=\Lambda L$ and a global action $\psi: \tilde{G} \times M \rightarrow M$ such that $\psi^{+}(L(\tilde{G}))=L, \psi^{+}$is a continuous linear map into $L$, and for $C \in \Lambda L ; \psi^{+}(C)=C(1)$.

Proof : By theorem (3.1). there exists a Lie group with Lie algebra $\Lambda \mathrm{L}$. Let. $\tilde{G}$ be the universal covering group (see Bourbaki [2, p.113]) of this group; then $\mathrm{L}(\tilde{\mathrm{G}})=\Lambda \mathrm{L}$. We have an infinitesimal left action, which we $\operatorname{cal1} \psi^{+}$, of $\tilde{G}$ on $M$ given by the following sequence of vector bundle morphisms,

$$
\begin{aligned}
& \Lambda \mathrm{L} \times \mathrm{M} \longrightarrow \mathrm{L} \times \mathrm{M} \stackrel{\mathrm{ev}}{\longrightarrow} \mathrm{T}(\mathrm{M}) \\
& (\mathrm{C}, \mathrm{p}) \longrightarrow(\mathrm{C}(1), \mathrm{p}) \longrightarrow \mathrm{C}(1)(\mathrm{p})
\end{aligned}
$$

where ev is the evaluation map which is a vector bundle morphism by condition (B1). The map $\psi^{+}: \mathrm{C} \longrightarrow \mathrm{C}(1)$ is continuous by theorem (3.1). The existence of the global action $\psi$ will follow from theorem (8.3) by showing that $\psi^{+}$is a uniform infinitesimal left action. 
By condition (B1), ev $: \mathrm{L} \times \mathrm{M} \longrightarrow \mathrm{T}(\mathrm{M})$ is a vector bundle morphism. Then the global version of the existence theorem for differential equations depending on a parameter (in this case the parameter space is $L$ ) implies that the map $(t, X, p) \longrightarrow \operatorname{Exp}(t X)(p)$ from $\mathbb{R} \times L \times M$ into $M$ is a morphism. The fact that this flow is defined on all of $\mathbb{R}$ follows from condition (A) .

Now let $B_{\rho}(0)$ be an open ball about 0 in $\Lambda \mathrm{L}$ on which $\exp _{\tilde{G}}$ is a diffeomorphism. We will show that $\psi^{+}$is uniform on $V=\exp _{\tilde{G}}\left(B_{\rho}(0)\right)$. Define, for each $\mathrm{p} \in \mathrm{M}$, the map $\delta^{\mathrm{p}}: \mathrm{g} \rightarrow\left(\mathrm{g}, \operatorname{Exp}\left(\psi^{+}\left(\exp _{\tilde{\mathrm{G}}}^{-1}(\mathrm{~g})\right)\right)(\mathrm{p})\right)$ from $V$ into $\tilde{G} \times M$. Let $\Sigma_{\mathrm{p}}$ be the maximal connected leaf containing (e, p) in the foliation $Y$ of $\tilde{G} \times M$ defined by the infinitesimal graph of $\psi^{+}$. For $X \in \mathcal{L}(G)$ and $P \in M$ define

$$
\alpha_{X}^{p}: t \rightarrow\left(\exp _{\tilde{G}}(t X), \operatorname{Exp}\left(t \psi^{+}(X)(p)\right)\right.
$$

from $\mathbb{R}$ into $\tilde{G} \times \mathrm{M}$. Now

$$
\begin{aligned}
T\left(\alpha_{X}^{p}\right)\left(\left.\frac{d}{d t}\right|_{S}\right) & =\left.\frac{d \alpha}{d t}\right|_{S} ^{p} \\
& =\left(\left.\frac{d}{d t}\right|_{t=0} \exp _{\tilde{G}}(t X) \exp _{\tilde{G}}(s X),\left.\frac{d}{d t}\right|_{t=s} \operatorname{Exp}\left(t \psi^{+}(X)\right)(p)\right) \\
& =\left(X\left(\exp _{\tilde{G}}(s X), \psi^{+}(X)\left(\operatorname{Exp}^{+}\left(s \psi^{+}(x)\right)(p)\right)\right)\right.
\end{aligned}
$$

belongs to $T(\tilde{G} \times M, Y)$. Then prop. (1.4) implies that the image of $\alpha_{X}^{p}$ is in $\Sigma_{p}$ since $\alpha_{X}^{p}(0)=(e, p)$ and $\mathbb{R}$ is connected. In particular if 
$g=\exp _{\tilde{G}}(X)$ for $X \cdot \varepsilon \cdot B_{\rho}(0)$ then

$$
\begin{aligned}
\alpha_{X}^{p}(1) & =\left(\exp _{\tilde{G}}(X), \operatorname{Exp}\left(\psi^{+}(X)\right)(p)\right) \\
& =\left(g, \operatorname{Exp}\left(\psi^{+}\left(\exp _{\tilde{G}}^{-1}(g)\right)(p)\right)\right.
\end{aligned}
$$

which shows that $\delta^{\mathrm{P}}(\mathrm{V}) \subset \Sigma_{\mathrm{p}}$.

Denote by $\mathrm{V}^{\mathrm{p}}$ the image of $\mathrm{V}$ under $\delta^{\mathrm{p}}$. The "projection" $\pi_{\tilde{G}}: Y \longrightarrow \tilde{G}$ obviously maps $\mathrm{V}^{\mathrm{p}}$ one-to-one onto $\mathrm{V}$. In fact we now show that $V^{p}$ is the component containing (e, p) in $\pi \tilde{G}(V) \cap \Sigma_{p}$. We have $(e, p)=\delta^{p}(e) \varepsilon V^{p}$. Suppose $(g, q)$ is any point in $V^{p}$ and let $U$ be an open set in $\Sigma_{p}$ containing $(g, q)$ on which $\pi_{\tilde{G}}$ is a diffeomoephism (prop. (6.3)). Let $W$ be an open set in $V \cap \pi \tilde{G}(U)$ containing ${ }^{\prime}$, then $\pi_{\tilde{G}}^{-1}: \pi_{\tilde{G}}(U) \rightarrow U$ takes $W$ onto an open set containing $(g, q)$ and $\left.\pi_{\tilde{G}}^{-1}\right|_{\pi_{\tilde{G}}(U)}(W) \subset V^{p} \quad$ since $\pi_{\tilde{G}}^{-1}=\delta^{p}$ on $W$. This proves that $V^{P}$. is open. In order to show that $\mathrm{V}^{\mathrm{P}}$ is the component containing $(\mathrm{e}, \mathrm{p})$ in $\pi_{\tilde{G}}^{-1}(V) \cap \Sigma_{p}$ it remains to prove that $V^{p}$ is closed in $\pi_{\tilde{G}}^{-1}(V) \cdot$ Let $(h, m)$ be any point in $\pi_{\tilde{G}}^{-1}(V) \cap \Sigma_{p}$ such that $(h, m) \notin v^{p}$. Now since $\pi_{\tilde{G}}$ is one-to-one on $V^{\mathrm{P}}$ there exists a unique point in $\mathrm{V}^{\mathrm{P}}$ with first component $h$, say $(h, n) . \Sigma_{p}$. is Hausdorff since it is an open submanifold of $Y$ and therefore we can find disjoint open neighbourhoods $A$ and $B$ of $(h, m)$ and $(h, n)$ respectively which $\pi_{\tilde{G}}$ maps diffeomorphically onto the same neighbourhood of $h$; this is possible since $\pi_{\tilde{G}}$ is a local. diffeomorphism. By restricting $A$ and. $B$ further we can assume that $\mathrm{A} \subset \mathrm{V}^{\mathrm{P}}$ and it then follows that $\mathrm{B} \cap \mathrm{V}^{\mathrm{P}}=\emptyset$ since $\pi \tilde{G}$ is one-to-one on 
$\mathrm{V}^{\mathrm{p}}$. This completes the proof that $\psi^{+}$is uniform and proves the theorem.

\section{Proof of Theorem $(10.2)$}

We keep the notations used above. I is assumed to be a subalgebra of $V(M)$ satisfying conditions (A) and (B):

Consider the idea1 ker $\psi^{+}=\{C \varepsilon L(\tilde{G}): C(1)=0\}$, in $L(\tilde{G})$ which is the kernel of the map $\psi^{+}: L(\tilde{G}) \rightarrow V(M)$; it is closed in $L(\tilde{G})$ since $\operatorname{ker} \psi^{+}=\bigcap_{\mathrm{p} \varepsilon \mathrm{M}}\left(\psi_{\mathrm{p}}^{+}\right)^{-1}\left(0_{\mathrm{p}}\right)$ where $\psi_{\mathrm{p}}^{+}$is the continuous linear map $X \rightarrow \psi^{+}(X)(p)$ from $L(\tilde{G})$ into $T_{p}(M) \cdot c_{p}$ denotes the zero vector in $\mathrm{T}_{\mathrm{p}}(\mathrm{M})$ ). Therefore $\operatorname{ker} \psi^{+}$is a Banach Lie subalgebra of $\mathrm{L}(\mathrm{G})$. Let

$$
L^{\prime}=\{C \varepsilon L(\tilde{G})=\Lambda L: C(t)=t X \text { for some } \cdots X \varepsilon \cdot L\} \text {, }
$$

then $L^{\prime}$ is a closed vector subspace in $L(\tilde{G})$ which complements $\operatorname{ker} \psi^{+}$, i.e. $\operatorname{ker} \psi^{+}$splits in $L(\tilde{G})$ and we identify $L(\tilde{G})$ with $\operatorname{ker} \psi^{+} \times \mathrm{L}^{\prime}$.

For $g \in \tilde{G}$, denote by $\psi_{g}$ the diffeomorphism $\mathrm{p} \rightarrow \psi(\mathrm{g}, \mathrm{p})$ of $\mathrm{M}$ into $\dot{\mathrm{M}}$. Let $\delta: \tilde{G} \longrightarrow \mathrm{D}(\mathrm{M})$ be the group homomorphism $\mathrm{g} \longrightarrow \psi_{\mathrm{g}}$. Let $\mathrm{H}=\operatorname{ker} \delta$, then we have a group isomorphism $\bar{\delta}: \tilde{G} / \mathrm{H} \longrightarrow \delta(\tilde{G})$. We will show that $H$ is a Lie subgroup of $\tilde{G}$. Condition (B2) gives the existence of a open neighbourhood $N$ of 0 in $L$ on which $\operatorname{Exp}$ is injective. Let $A \times B \subset \operatorname{ker} \psi^{+} \times L^{\prime}=L(\tilde{G})$ be an open neighbourhood of 0 on which $\exp _{\tilde{G}}$ is a diffeomorphism and such that $\psi^{+}(\mathrm{A} \times \mathrm{B}) \subset \mathrm{N}$. Let $\mathrm{h} \varepsilon \mathrm{H} \cap \exp _{\tilde{\mathrm{G}}}(\mathrm{A} \times \mathrm{B})$, then $h=\exp _{\tilde{G}}(C)$ for some unique $C \varepsilon A \times B$. Now for all $p \varepsilon M$ we have 


$$
\begin{aligned}
\mathrm{p}=\psi(\mathrm{h}, \mathrm{p}) & =\psi\left(\exp _{\tilde{\mathrm{G}}}(\mathrm{C}), \mathrm{p}\right) \\
& \left.=\operatorname{Exp}\left(\psi^{+}(\mathrm{C})\right)(\mathrm{p}) \quad \text { (by prop. }(8.4)\right) \\
& =\operatorname{Exp}(\mathrm{C}(1))(\mathrm{p}) .
\end{aligned}
$$

Since $C(1) \in N$ and $\operatorname{Exp}(C(1))=$ id we have $C(1)=0$, i.e. $C \varepsilon A \times\{0\}$. Also if $C \varepsilon A \times\{0\}$ then $\psi(\exp (C), p)=p$ for all $p$ and $\exp (C) \varepsilon H$. The fact that $\mathrm{H}$ is a lie subgroup then follows from prop. (3.3) since $\exp _{\tilde{G}}(A \times\{0\})=H \cap \exp _{\tilde{G}}(A \times B)$. We alșo have $L(H)=\operatorname{ker} \psi^{+}$.

It follows from prop. (3.6) that there exists a connected Lie group structure on $\tilde{G} / H$ such that the projection $p: \tilde{G} \rightarrow \tilde{G} / H$ is a submersion and $\check{\mathrm{L}}(\tilde{\mathrm{G}} / \mathrm{H}) \stackrel{\dot{ }}{=} \mathrm{L}(\tilde{\mathrm{G}}) / \mathrm{L}(\mathrm{H}) \cong \mathrm{L}$. Using the group isomorphism $\bar{\delta}: \tilde{G} / \mathrm{H} \longrightarrow \delta(\tilde{G})$ we have a Lie group structure induced on $\delta(\tilde{G})$ such that $\delta$ is a submersion, $\operatorname{ker} L(\delta)=\operatorname{ker} \psi^{+}, L(\delta(\tilde{G})) \cong L$, and $\bar{\delta}$ is a diffeomorphism. With this Lie group structure $\delta(\tilde{G})$ will be denoted by $G$. Define $\phi: G \times M \longrightarrow M$ to be the natural action $\phi(g, p)=g(p)$. Let $\alpha$ be the submersion $(k, p) \longrightarrow(\delta(k), p)$ of $\tilde{G} \times M$ into $G \times M$. Then $\psi=\phi \circ \alpha$ and $\phi$ is a morphism since $\psi$. is a morphism and $\alpha$ is a submersion.

We now show that $\phi^{+}$is a Banach Lie algebra isomorphism. Let $\mathrm{C} \varepsilon \mathrm{L}(\tilde{\mathrm{G}})=\Lambda \mathrm{L}$ and $\mathrm{p} \in \mathrm{M}$, then

$$
\begin{aligned}
C(1)(p) & =\psi^{+}(C)(p) \\
& =T_{(e, p)} \psi\left(C(e), 0_{p}\right)
\end{aligned}
$$




$$
\begin{aligned}
& =T_{(e, p)}(\phi \circ \alpha)\left(C(e), 0_{p}\right) \\
& =T_{(j . d, p)}{ }^{\left(T_{e} \delta(C(e)), O_{p}\right)} \\
& =\phi^{+}(L(\delta)(C))(p), \\
\text { i.e. } \psi^{+} & =\phi^{+} \text {oL }(\delta) .
\end{aligned}
$$

We see that $\phi^{+}$maps $L(G)$ onto $L$ since $\psi^{+}$maps $L(\tilde{G})$ onto $L$ (theorem $(10.3))$. Since $L(\delta)\left(\right.$ ker $\left.\psi^{+}\right)=0$ we have that $\phi^{+}$is injective. The fact that $\phi^{+}$is continuous follows easily from the following;

(I) $\psi^{+}$is continuous

(2) $L(\delta)$ is continuous, surjective and $\operatorname{ker} \ddot{L}(\delta)=\operatorname{ker} \psi^{+}$ splits in $\mathrm{L}(\tilde{\mathrm{G}})$ (since $\delta$ is a submersion).

It remains to prove the uniqueness of $G$. Let $F$ be another Lie transformation group with the same properties as $G$ and let $\beta: F \times M \rightarrow M$ be the $\operatorname{map}(f, p) \rightarrow f(p)$. Now $\exp _{F}(t X)=\operatorname{Exp}\left(t \beta^{+}(X)\right)$ for $X \varepsilon L(F)$ by prop. ( 9.1 ) and therefore since $F$ is connected it is generated by Exp (L). Similarly $G$ is generated by Exp (L) which shows that the underlying groups of $G$ and $F$ are the same in $D(M)$. The following commutative diagram

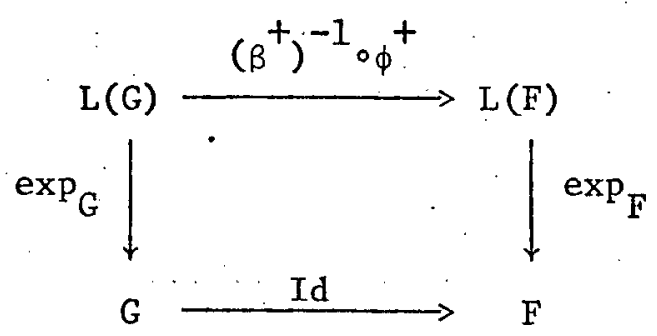


shows that Id from $G$ into $F$ is a morphism and completes the proof of the theorem.

\$11: A Banach Lie algebra of complete vector fields which does not generate a connected Lie transformation group

If we have a subalgebra $L$ of $V(M)$ which satisfies condition (A) and admits a Banach Lie algebra structure such that (B1) is true but not (B2) then prop. (9.4) implies that this. I won't admit any Banach space structure such that $(B 2)$ is satisfied. Hence by prop. (9.5), L isn't the image of the infinitesimal generator of any connected Lie transformation group.

We now give an example of such an $L$ which, although it doesn't generate a connected Lie transformation group, is still enlargeable. Let $M=\operatorname{disjoint} \bigcup_{n=1}^{\infty} S_{n}^{1}$ where $S_{n}^{1}$ is the unit circle $S^{1}$. Define the vector field $x_{n}$ by

$$
X_{n}(p)= \begin{cases}0 & \text { if } p \in s_{j}^{1} \text { and } j \neq n \\ \frac{d \alpha}{d t}(p) & \text { if } p \in s_{n}^{1}\end{cases}
$$

where $\alpha$ is the curve on $s_{n}^{1} ; t \rightarrow e^{2 \pi i t}$

Let $L$ be the normed vector space consisting of all sums $\sum_{n=1}^{\infty} c_{n} x_{n}, \quad c_{n} \in \mathbb{R}$, such that $\sum_{n=1}^{\infty} \frac{\left|c_{n}\right|}{n}<\infty$. If $c=\sum c_{n} x_{n} \varepsilon L$, define 
the norm of $C$ to be $\|C\|=\sum_{n=1}^{\infty} \frac{\left|c_{n}\right|}{n}$. We will show that $L$ is a Banach Lie algebra satisfying (A) and (B1) but not (B2). L consists of complete vector fields since we have a disjoint union of compact manifolds. It is a vector space for

$$
\begin{aligned}
\left\|\sum c_{n} x_{n}+\sum b_{n} x_{n}\right\| & =\sum \frac{\left|c_{n}+b_{n}\right|}{n} \\
& \leq \sum \frac{\left|c_{n}\right|}{n}+\sum \frac{\left|b_{n}\right|}{n}<\infty
\end{aligned}
$$

if $\sum c_{n} x_{n}$ and $\sum b_{n} x_{n}$ belong to L. Similarly $L$ is closed under scalar multiplication. $L$ is closed under the bracket operation since

$$
\left[\sum c_{n} x_{n},\left[b_{n} x_{n}\right]=\sum_{i, j} c_{i} b_{j}\left[x_{i}, x_{j}\right]=0\right.
$$

We now give the usual proof that a space of sequences is complete. Let $\left\{A^{n}\right\}$ be a Cauchy sequence of elements in $L$, i.e. given $\varepsilon>0$ there exists $N$ such that if $i, j>N$ we have

$$
\left\|A^{i}-A^{j}\right\|=\sum_{k} \frac{\left|A_{k}^{i}-A_{k}^{j}\right|}{k}<\varepsilon
$$

where $A^{n}=\sum_{k} A_{k}^{n} X_{k}$. In particular this implies that for fixed $k,\left\{A_{k}^{i}\right\}$ is a Cauchy sequence. Let $A_{k}=\lim _{i \rightarrow \infty} A_{k}^{i}$ and $A=\sum A_{k} X_{k}$. We will show that $A \in L$ and $\lim _{n \rightarrow \infty} A^{n}=A$. From above we have

$$
\sum_{k=1}^{s} \frac{\left|A_{k}^{i}-A_{k}^{j}\right|}{k}<\varepsilon
$$


for all $s \geq 1$ and $i, j>N$, then

$$
\lim _{i \rightarrow \infty} \sum_{k=1}^{s} \frac{\left|A_{k}^{i}-A_{k}^{j}\right|}{k}=\sum_{i=1}^{s} \frac{\left|A_{k}-A_{k}^{j}\right|}{k}<\infty \quad \text { for al1 } s>1
$$

Since this is true for all $s$ we have

$$
\sum_{k=1}^{\infty} \frac{\left|A_{k}-A_{k}^{j}\right|}{k}<\varepsilon
$$

But this implies that ${ }^{\prime} A-A^{j} \varepsilon L$ which means $A=\left(A-A^{j}\right)+A^{j} \varepsilon L$. We also have for $j>N,|| A-A^{j}||<\varepsilon$; which shows that $\lim A^{j}=A$. We have now shown that $L$ is a Banach. Lie algebra and it is trivially enlargeable since it is abelian.

It remains to show that the evaluation map ev $: \mathrm{L} \times \mathrm{M} \rightarrow \mathrm{T}(\mathrm{M})$ is a vector bundle morphism. Local coordinates on each of the $\mathrm{S}_{\mathrm{n}}^{1}$ 's are given by the local inverse of the map $t \rightarrow e^{2 \pi i t}$. We denote this map by $\log _{\mathrm{n}}$. This induces local coordinates on $\mathrm{T}(\mathrm{M})$ and we denote this map by $\log _{n}$. If $\pi: T(M) \rightarrow M$ is the usual projection and if $Z=\sum z_{n} X_{n}$ then

$$
\log _{n}^{T}(Z(p))=\left(\log _{n}\left(\pi(Z(p)), z_{n}\right)=\left(\log _{n}(p), z_{n}\right)\right.
$$

Denote by $\pi_{n}$ ' the continuous linear map from $L$ into $R$ given by $\sum a_{k} X_{k} \rightarrow a_{n}$. A local coordinates map at $(Y, P) \in L \times M$ is given by $(Y, p) \longrightarrow\left(Y, \log _{n}(p)\right)$ if. $p \in S_{n}^{\prime}$. Let $Y=\sum y_{k} X_{k}$ and $W$ be an open neighbourhood of $Y(p)=e v(Y, p)$ in $T(M)$. Now $\log _{n}^{T}(Y(p))=\left(\log _{n}(p), y_{n}\right)$ 
and by the definition of the topology of $T(M)$ there exists an open set $\mathrm{U}$ containing $\log _{\mathrm{n}}(\mathrm{p})$ and an interval : $\mathrm{B}_{\rho}\left(\mathrm{y}_{\mathrm{n}}\right)$ about $\mathrm{y}_{\mathrm{n}}$ such that $\left(\log _{n}^{T}\right)^{-1}\left(U \times B_{\rho}\left(y_{n}\right)\right) \subset W$. Let $z=\sum z_{n} x_{n}$, if $(Z, q) \varepsilon B_{\rho} / \mathrm{n}(\mathrm{Y}) \times\left(\log _{\mathrm{n}}\right)^{-1}(\mathrm{U}) \subset \mathrm{L} \times \mathrm{M}$, then $\|\mathrm{Z}-\mathrm{Y}\|<\rho / \mathrm{n}$ which

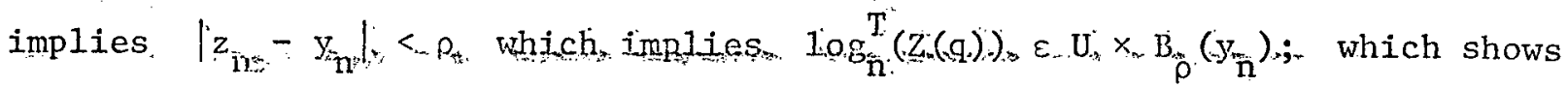
$\operatorname{ev}\left(B_{\rho / n}(Y) \times U\right) \subset W$. Hence ev. is continuous and these local coordinates ev. is given by the niap. $\tau$ in the following diagram,

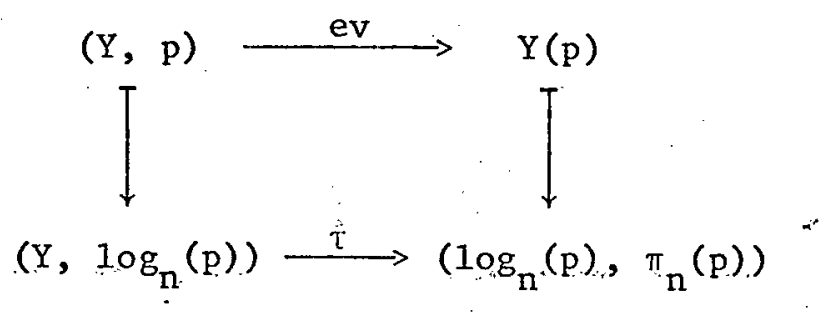

and $\tau$ is a morphism since $\pi_{n}$ is. This proves that ev is a morphism of manifolds. It is a vector bundle morphism since the constant map $p \rightarrow \pi_{n}$ is a morphism from $U$ into the space of continuous linear maps from $L$ into $\mathbb{R}$.

Condition (B2) doesn't hold because Exp doesn't have a radius of injectivity at 0 in $\dot{L}$, for $\operatorname{Exp} X_{n}=$ identity for all $n$ and $\lim _{n \rightarrow \infty} x_{n}=0$

Although $L$ doesn't generate a connected Lie transformation group theorem (10.3) ensures that.it is the image of the infinitesimal generator of a global left action. 


\section{Bibliography}

[1] N. Bourbaki, Variêtés différentialles et analytiques, Hermann, Paris, 1971.

[2] N. Bourbaki, Groupes et algèbres de Lie, Hermann, Paris, 1972, chapters 2 and 3

[3] H. Chu and S. Kobayashi, the automorphism group of a geometric structure, Trans. Amer. Math. Soc. 113 (1964) pp. 141-150.

[4] H.T. van Est and Th. J. Korthagen, Non-enlargeable Lie algebras, Indag. Math. 26. (1964): pp. 15-31.

[5] S. Lang, Differentiable Manifolds, Addison-Wesley; Reading; Mass., 1972.

[6] 0. Loos, Lie transformation groups of Banach manifolds, Journal of Differential Geometry 5 (1971) pp. 175-185.

[7] R.S. Palais, A global formulation of the Lie theory of transformation groups, Memoirs of the Amer. Math. Soc. No. 22, 1957.

[8] S. Swierczkowski, The path-functor on Banach Lie algebras, Proc. Kon. Ned. Akad. v. Weterrsch., Amsterdam, 74 (1971) pp. 235-239. 